\title{
A common developmental plan for neocortical gene-expressing neurons in the pallium of the domestic chicken Gallus gallus domesticus and the Chinese softshell turtle Pelodiscus sinensis
}

\author{
Ikuo K. Suzuki ${ }^{1,2}{ }^{*}$ and Tatsumi Hirata ${ }^{1}$ * \\ ${ }^{1}$ Division of Brain Function, National Institute of Genetics, Graduate University for Advanced Studies (Sokendai), Mishima, Japan \\ ${ }^{2}$ Institute of Interdisciplinary Research in Human and Molecular Biology, Université Libre de Bruxelles, Brussels, Belgium
}

\section{Edited by:}

Ryuichi Shigemoto, National Institute for Physiological Sciences, Japan

\section{Reviewed by:}

Alino Martinez-Marcos, Universidad de Castilla, Spain

Christoph Redies, University of Jena

School of Medicine, Germany

\section{${ }^{*}$ Correspondence:}

Ikuo K. Suzuki, Institute of

Interdisciplinary Research in Human and Molecular Biology, Université

Libre de Bruxelles, Brussels B-1070,

Belgium

e-mail: suzuki.ikuo@gmail.com

Tatsumi Hirata, Division of Brain Function, National Institute of

Genetics, Graduate University for Advanced Studies (Sokendai), Yata 1111, Mishima 411-8540, Japan e-mail: tathirat@lab.nig.ac.jp
The six-layered neocortex is a unique characteristic of mammals and likely provides the neural basis of their sophisticated cognitive abilities. Although all mammalian species share the layered structure of the neocortex, the sauropsids exhibit an entirely different cytoarchitecture of the corresponding pallial region. Our previous gene expression study revealed that the chicken pallium possesses neural subtypes that express orthologs of layer-specific genes of the mammalian neocortex. To understand the evolutionary steps leading toward animal group-specific neuronal arrangements in the pallium in the course of amniote diversification, we examined expression patterns of the same orthologs and a few additional genes in the pallial development of the Chinese softshell turtle Pelodiscus sinensis, and compared these patterns to those of the chicken. Our analyses highlighted similarities in neuronal arrangements between the two species; the mammalian layer 5 marker orthologs are expressed in the medial domain and the layer 2/3 marker orthologs are expressed in the lateral domain in the pallia of both species. We hypothesize that the mediolateral arrangement of the neocortical layer-specific gene-expressing neurons originated in their common ancestor and is conserved among all sauropsid groups, whereas the neuronal arrangement within the pallium could have highly diversified independently in the mammalian lineage.

Keywords: neocortex, neuron type, pallium, turtle, evolution

\section{INTRODUCTION}

Complex cognitive functions in mammalian species are essentially encoded in the neural circuits of the neocortex, a mammalianspecific structure characterized by tangential neuronal layers and located inside the pallium (the dorsal part of the telencephalon). In each layer, excitatory neurons with similar phenotypes are tangentially arranged. For example, extracortically projecting neurons reside in the deep layers 5 and 6 , whereas the majority of intracortically connecting neurons are located in the more shallow layer 2/3 (Molyneaux et al., 2007). This laminar neuronal arrangement is basically shared by all studied mammalian species, including even the monotremes, and marsupials (Butler and Hodos, 2005). In contrast, the sauropsids, which is a group containing the currently living reptiles and birds, possess a totally different neuronal arrangement in the corresponding pallial region to the mammalian neocortex (Figure 1). This structural difference in neocortical regions has again raised the longstanding question of how mammals acquired the layered neocortex during evolution.

In mammalian neocortical development, layer-specific neuron subtypes differentiate from multipotent neural progenitors residing in the ventricular zone (VZ) of the pallium (Temple, 2001). Depending on the timing of their generation, newly generated neurons express transcription factors that provide layer-specific characteristics (Molyneaux et al., 2007; Greig et al., 2013). For example, layer 5 neurons generated earlier express Fezf2 and its downstream target Ctip2, which are required and sufficient for their axons to project to extracortical targets (Arlotta et al., 2005; Chen et al., 2005, 2008; Molyneaux et al., 2005). In contrast, layer $2 / 3$ neurons generated later express Satb2, a transcriptional repressor of Ctip2, and thereby project to intracortical targets by suppressing Ctip2-driven extracortical projections (Alcamo et al., 2008; Britanova et al., 2008). The fate-determining role of these transcription factors makes them ideal functional markers for layer-specific neuronal subtypes.

We, and other research groups, have recently found that the avian pallium houses neuron subtypes sharing the molecular expression, and axon projection patterns of layer-specific neuron subtypes in the mammalian neocortex (Dugas-Ford et al., 2012; Suzuki et al., 2012; Chen et al., 2013; Jarvis et al., 2013). In particular, the above-mentioned layer 5 transcription factors are expressed by neurons that occupy the medial domain in the pallium of chicks. On the other hand, the layer 2/3-specific transcription factors are expressed by neurons in the lateral domain (Suzuki and Hirata, 2012; Suzuki et al., 2012). These observations suggest that the pallia of sauropsids contain distinct neuronal populations that 


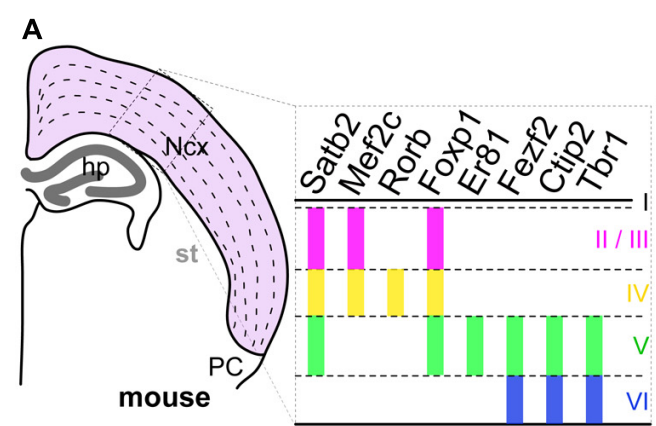

FIGURE 1 | Comparison of pallial structures among three amniotes. The pallial subdivisions of the mouse (A), chicken (B), and turtle (C) are drawn based on their cytological characteristics. The mouse neocortex and its corresponding pallial regions in the chicken and turtle are colored in pink. The distinctive dense cellular layer in the turtle pallium is indicated by the thick dark-gray line. The right panel in $(\mathbf{A})$ represents expression patterns of

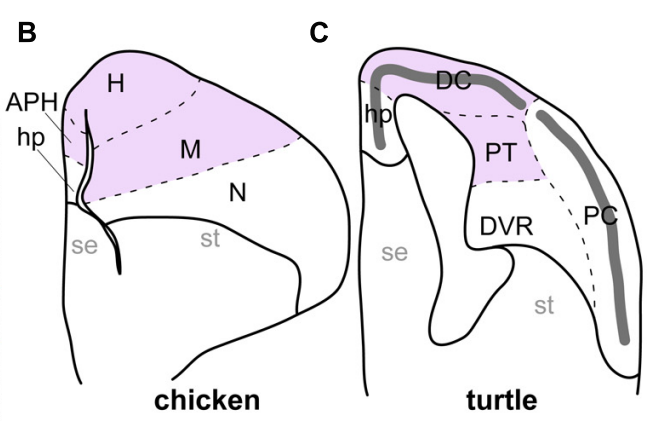

layer-specific transcription factor genes in the mammalian neocortex. Colored vertical bars indicate the layers in which each layer-specific marker gene is expressed. Abbreviations: APH, parahippocampal region; DC, dorsal cortex; DVR, dorsal ventricular ridge; $H$, hyperpallium; hp, hippocampus; $M$, mesopallium; N, nidopallium; Ncx, neocortex; PC, piriform cortex; PT, pallial thickening; se, septum; st, striatum. have characteristics similar to mammalian neocortical neurons, and raise an important question about the evolutionary changes of neuronal arrangements in the amniote pallia. What remained unclear was the generality of these findings among diverse amniote groups, because we only had limited knowledge of the neuronal arrangement in two distantly related animal groups: mammals and birds.

On the basis of our current understanding of amniote phylogeny, the lineages leading to the living mammals and the sauropsids diverged around 316 million years ago (Wang et al., 2013). Subsequently, the early sauropsids further diverged into several distinct reptilian groups, including the turtles. More recently, the birds originated from a reptilian group including the dinosaurs (Carroll, 1988; Kumar and Hedges, 1998; Hedges et al., 2006; Wang et al., 2013). Amniote pallia are histologically classified into three different types (Figure 1; Northcutt and Kaas, 1995; Medina and Reiner, 2000; Aboitiz and Zamorano, 2013). The first type has the six-layered cytoarchitecture only found in the mammalian neocortex (Figure 1A). The second has the domain architecture consisting of multiple nuclei commonly observed in birds (Figure 1B). The last is a simpler type found in non-avian reptiles such as lizards and turtles, in which a single neuronal layer spans the whole pallium (Figure 1C). Because it has the simplest cytoarchitecture, the pallium of the non-avian reptiles is considered as having retained the ancient state of the amniote common ancestor, from which the more complexly structured pallia in birds, and mammals have independently evolved (Marin-padilla, 1978; Medina and Reiner, 2000). However, despite its evolutionarily important position, gene expression in the reptilian pallium has been characterized in only a few studies (Dugas-Ford et al., 2012; Nomura et al., 2013a).

The current study describes comparative developmental expressions of gene orthologs of neocortical layer-specific transcription factors in the pallium of the Chinese softshell turtle Pelodiscus sinensis. We chose this turtle species because of the turtles' phylogenetic position in the sauropsids, with a sister relationship to the birds (Kumazawa and Nishida, 1997; Iwabe et al., 2004; Wang et al., 2013). Even though small structural variations exist among reptile pallia (Aboitiz, 1999), this species has a pallium that represents the typical simple cytoarchitecture of non-avian reptiles well (Figure 1C). Furthermore, the commercial availability of fertilized eggs and accumulated knowledge about its embryonic development make this species an excellent model for detailed developmental research (Tokita and Kuratani, 2001; Nomura etal., 2013b). We examined the expression patterns of eight transcription factors whose layer-specific expression in the mammalian neocortex is implicated in the layer-specific characteristics of neurons (Molyneaux et al., 2007). We also advanced the characterization of gene expressions in the chick pallium beyond that achieved in previous studies by using other layer markers and differently staged embryos. On the basis of these results, we compare the differentiation and arrangement of neuron subtypes that express these genes in the pallia of different amniote groups, and discuss the evolutionary aspects of pallial organization.

\section{MATERIALS AND METHODS ANIMALS}

Fertilized turtle and chicken eggs were purchased from local farmers, Daiwa Yoshoku (Oita, Japan) and Ohata Shaver (Shizuoka, Japan), respectively. We used 15 turtle and 15 chicken eggs for this study. The sexes of animals were uncertain. The day on which the eggs were transferred to $30^{\circ} \mathrm{C}$ (turtle) or $37^{\circ} \mathrm{C}$ (chicken) was designated as embryonic day 0 (E0). Developmental stages of the turtle and chicken embryos were defined according to previous studies (Hamburger and Hamilton, 1951; Tokita and Kuratani, 2001) and designated as TK and $\mathrm{HH}$, respectively. All experimental protocols were approved by the Animal Committee of the National Institute of Genetics and carried out according to their guidelines.

\section{cDNA CLONING}

The cDNA fragments coding for turtle Satb2, Mef2c, Fezf2, Er81, Rorb, and Tbr 1 were amplified by RT-PCR with degenerate primers (Table 1) and subcloned into the plasmid vector pTA2 (TOYOBO, Tokyo, Japan). The cDNA fragment for chicken Rorb was similarly isolated using specific primers (Table 1). The other chicken genes 
used in the study were isolated in previous studies (Puelles et al., 2000; Sugiyama and Nakamura, 2003; Suzuki et al., 2012) and verified to contain the specified range of nucleotide sequences (Table 1) of the genes as annotated in the public databases.

\section{DNA SEOUENCE ANALYSIS}

Cloned genes were sequenced using a multi-capillary sequencer (ABI Prism 3130; Life Technologies, Carlsbad, CA, USA) and compared with the sequences of homologous genes obtained from the NCBI and ENSEMBL databases. The multiple sequence alignment was generated and edited using MEGA5 software (Tamura et al., 2011). Molecular phylogenetic trees were constructed by the neighbor joining method (Saitou and Nei, 1987) using MEGA5, and drawn with FigTree software (http://tree.bio.ed.ac.uk/ software/figtree/).

\section{IN SITU HYBRIDIZATION}

Brains of turtle and chick embryos were fixed with $4 \%$ paraformaldehyde in phosphate-buffered saline (PBS) overnight at $4^{\circ} \mathrm{C}$ and processed for coronal frozen sections. In situ hybridization was performed as previously described (Suzuki et al., 2012). Briefly, the sections were soaked with methanol at $-30^{\circ} \mathrm{C}$ for $15 \mathrm{~min}$, treated with $25 \mu \mathrm{g} / \mathrm{ml}$ proteinase $\mathrm{K}$ at room temperature for $5 \mathrm{~min}$, and hybridized with $1 \mu \mathrm{g} / \mathrm{mL}$ DIG-labeled antisense RNA probes in hybridization buffer (50\% formamide, $1 \times$ Denhardt [Am-34-resco], $0.25 \mathrm{mg} / \mathrm{mL}$ RNA [Roche], $1 \times \mathrm{PE}$, $100 \mu \mathrm{g} / \mathrm{mL}$ heparin, $0.1 \%$ Tween $20,0.75 \mathrm{M} \mathrm{NaCl}$ ) at $60^{\circ} \mathrm{C}$ overnight. The probes were synthesized using the isolated cDNA clones (Table 2) as the templates. After washing, the hybridization signals were detected with anti-DIG antibody conjugated with alkaline phosphatase (Roche, Basel, Switzerland) and visualized with NBT/BCIP solution (Roche).

\section{IMMUNOHISTOCHEMISTRY}

Brain sections were prepared as described for in situ hybridization. The sections were washed with TBST (10 mM Tris pH7.4, $150 \mathrm{mM} \mathrm{NaCl}, 0.1 \%$ Tween20), permeablized with 100\% methanol at $-30^{\circ} \mathrm{C}$ for $15 \mathrm{~min}$, and then reacted with the primary antibodies at $4^{\circ} \mathrm{C}$ overnight. The following antibodies were used: mouse anti-Cadherin7 antibody [CCD7-1; Developmental Studies Hybridoma Bank (DSHB), Iowa, IA, USA], rat anti-Ctip2 antibody (ab18465; Abcam, Cambridge, UK), rabbit anti-Foxp1 antibody (ab16645; Abcam), mouse anti- $\beta$ (III)-tubulin antibody, TUJ1 (MMS-435P; Covance, Princeton, NJ, USA), and rabbit anti-phospho-Histone $\mathrm{H} 3$ antibody (06-570; Millipore, Billerica, MA, USA), After washing with TBST, the sections were stained with anti-mouse IgG antibody conjugated with Alexa Fluor 488 (A11029; Life Technologies), anti-rat IgG antibody

Table 1 | List of in situ probes used.

\begin{tabular}{|c|c|c|}
\hline Name & Target & Primers for amplification of gene fragment \\
\hline \multirow[t]{2}{*}{ psSatb2 } & Turtle Satb2 & 5'-TGCCAGGAGTTTGGGAGATGG-3' \\
\hline & & 5'-CTGTGTGCGRTTGAAWGCCAC-3' \\
\hline \multirow[t]{2}{*}{ psMef2c } & Turtle Mef2c & 5'-CCCACGCACTGAAGAAAAAT-3' \\
\hline & & 5'-TTGTYGARATGGCTGATGGRT-3' \\
\hline \multirow[t]{2}{*}{ psER81 } & Turtle Er81 & 5'-CARGARACATGGCTTGCHGA-3' \\
\hline & & 5'-ACTGGRTCRTGRTACTCCTG-3' \\
\hline \multirow[t]{2}{*}{ psFezf2 } & Turtle Fezf2 & 5'-GCGCAYTACAACCTSACSCGSC-3' \\
\hline & & 5'-GGCTTCTTGTCRTTGTGSGT-3' \\
\hline \multirow[t]{2}{*}{ psTbr1 } & Turtle Tbr1 & 5'-CARGACCAGTTCGTSAGCAA-3' \\
\hline & & 5'-CTGGAGTCGGACARGTCYTT-3' \\
\hline \multirow[t]{2}{*}{ psRorb } & Turtle Rorb & 5'-TCCARKCCAGACTGATCKGG-3' \\
\hline & & 5'-GCCGMCTGCAGAAGTGYCTKG-3' \\
\hline \multirow[t]{2}{*}{ cSatb2 } & Chicken Satb2 & 5'-ACCAGCACCCACAAGCTATCAACC-3' \\
\hline & (Nucleotide 2302-2915 of XM_421919) & 5'-ACTCСТССТСАTAGATCACАTСССТСТС-3' \\
\hline \multirow[t]{2}{*}{ cMef2c } & Chicken Mef2c & 5'-CGTTGAGAAAGAAAGGACTTAATGG-3' \\
\hline & (Nucleotide 886-1583 of XM_001231661) & 5'-CCATCAGCCATCTCAACAACATATGGTAC-3' \\
\hline \multirow[t]{2}{*}{ cER81 } & Chicken Er81 & Gift from Dr. Nakamura \\
\hline & (Nucleotide 1868-2787 of NM_204917) & Sugiyama and Nakamura (2003) \\
\hline \multirow[t]{2}{*}{ cFezf2 } & Chicken Fezf2 & 5'-CAAGAGCCTGGCCTTCTCCA-3' \\
\hline & (Nucleotide 280-1090 of XM_414411) & 5'-TGAGCGTGGAGCTCCTGTTG-3' \\
\hline \multirow[t]{2}{*}{ cTbr1 } & Chicken Tbr1 & Gift from Dr. Shimamura \\
\hline & (Nucleotide 4-2132 of XM_003641638 and poly A tail) & Puelles etal. (2000) \\
\hline \multirow[t]{2}{*}{ cRorb } & Chicken Rorb & 5'-GGTTTACAGCAACAGCATCAGCAAC-3' \\
\hline & (Nucleotide 682-1245 of XM_205093) & 5'-GCTTGGAAGTGGTTTTGGTGAGAATGTG-3' \\
\hline
\end{tabular}


Table 2 | Turtle cDNA fragments isolated.

\begin{tabular}{|c|c|c|c|}
\hline Name & Accession No. & Length (bp) & Top blast hit \\
\hline Satb2 & AB689003 ENSPSIT00000020487 & 521 & 92\% identical to chicken Satb2 (XM_421919) \\
\hline Mef2c & AB689005 ENSPSIT00000013823 & 601 & 93\% identical to Green Anole Mef2c (XM_003216358) \\
\hline Er81 & AB689006 ENSPSIT00000009495 & 547 & 93\% identical to chicken Er81 (NM_204917) \\
\hline Fezf2 & AB689007 ENSPSIT00000010810 & 387 & 90\% identical to chicken Fezf2 (XM_414411) \\
\hline Tbr1 & AB689004 ENSPSIT00000004703 & 532 & 87\% identical to chicken Tbr1 (XR_026840) \\
\hline Rorb & AB689002 ENSPSIT00000006123 & 319 & 90\% identical to Green Anole Rorb (XM_003216536) \\
\hline
\end{tabular}

conjugated with Alexa Fluor 488 (A11006; Life Technologies) or anti-rabbit IgG antibody conjugated with Cy3 (711-165-152; Jackson ImmunoResearch, West Grove, PA, USA). They were then washed with TBST again, and coverslipped with $90 \%$ glycerol in PBS.

\section{IMAGING}

Bright-field and fluorescence images were captured using a fluorescent microscope (Axioplan2; Zeiss, Jena, Germany) using a CCD camera (DP71; Olympus, Tokyo, Japan). The brightness and contrast of images were adjusted using Adobe Photoshop CS4 software (Adobe Systems; San Jose, CA, USA).

\section{RESULTS}

\section{PALLIAL SUBDIVISIONS IN THE TURTLE}

The matured turtle pallium has been cytologically subdivided into several domains in the previous literature (Johnston, 1915; Powers and Reiner, 1980; Goffinet, 1983). Briefly, the trilaminar domain covers the superficial part, and is further subdivided into the hippocampus, dorsal cortex (DC), and piriform cortex (PC) along the mediolateral axis (Figure 1C). These three subdivisions commonly have the cytological organization that contains a single layer of pyramidal neurons (Figure 2A). This layer is sandwiched between the exterior, axon-dense, and interior, axon-sparse, layers (Figure 2B) that also contain scattered interneurons (Blanton and Kriegstein, 1991). The pyramidal neurons in the single packed layer possibly consist of multiple neuronal populations that have distinct connection targets and molecular expressions, as shown in some other reptile species (Martínez-Marcos et al., 1999; Nomura et al., 2013a). Underneath the trilaminar domain are the nuclear domains that consist of the pallial thickening (PT) and dorsal ventricular ridge (DVR). The former is continuously extended ventrally from the DC, and the latter protrudes into the lateral ventricle from the most ventral part of the pallium (Figure 1C). Among these subdivisions of the turtle pallium, the DC, and PT are accepted as the homolog of the mammalian neocortex because of their shared features, including the expression of Emxl, and reciprocal connections with the thalamus (Heller and Ulinski, 1987; Fernandez et al., 1998; Reiner, 2000).

In contrast, the chick pallium does not have an apparent cellular layer. Until the nuclear structure manifests with clustered neurons by the end of the embryonic stages, neurons are more or less evenly scattered across the pallium (Figures 2D,E). The protrudent DVR in the chick pallium is anatomically subdivided

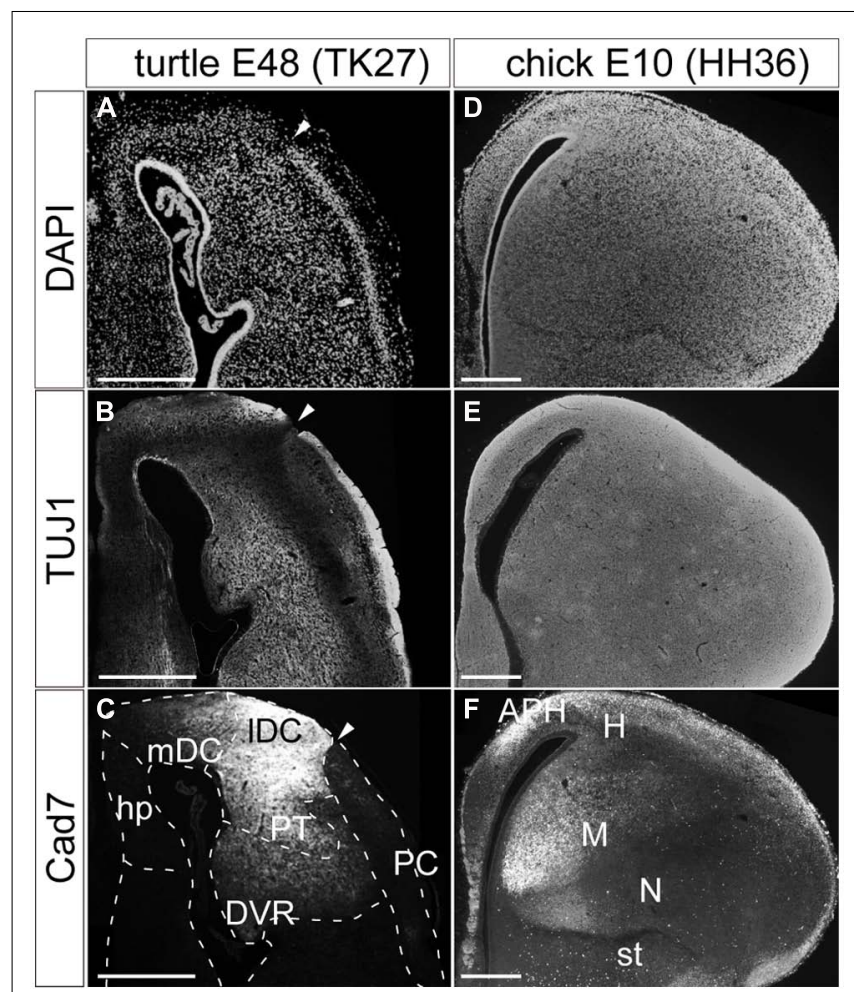

FIGURE 2 | Pallial subdivisions of the turtle and chick. Coronal sections of the turtle (A-C) and chick (D-F) pallia at E48, and at E10, respectively, in which the mediolateral axis runs left to right, and the dorsoventral axis runs top to bottom. (A,D) Nuclear staining by DAPI. (B,E) Immunostaining with TUJ1 antibody strongly labels axons in the turtle and chick pallia. (C,F) Immunostaining for Cad7. Note the strong specific labeling of the lateral part but not the medial part of the turtle DC and the chick mesopallium. White arrowheads indicate the boundary between the DC and PC in the turtle pallium. Scale bars: $250 \mu \mathrm{m}$. Abbreviations: Cad, cadherin; DVR, dorsal ventricular ridge; hp, hippocampus; IDC, lateral part of the dorsal cortex; mDC, medial part of the dorsal cortex; PC, piriform cortex; PT, pallial thickening.

into the dorsal mesopallium and ventral nidopallium. The former division and the hyperpallium are accepted as the Emx1-positive field homologous to the mammalian neocortex (Fernandez et al., 1998).

Expression of cadherin proteins, in particular Cad7, has been used to define the avian pallial subdivisions (Redies et al., 2001). In the developing chick pallium, Cad7 labeled the parahippocampal 
region (APH) and other restricted parts of the pallial divisions (Figure 2F). Of note is that its restricted expression further subdivides the pallium. For example, the medial parts of the mesopallium or nidopallium are compartmentalized by strong Cad7 expression. Likewise, the same antibody against Cad7 supported further subdivisions of the turtle pallium (Figure 2C). The protein was strongly expressed in parts of the DC and PT. In the DC, it was more strongly expressed in the lateral domain, clearly delineating the lateral from the medial DC. Thus, in a molecular context, the turtle DC seems divisible into medial and lateral compartments, although the two domains appeared cytologically homogeneous and continuous.

\section{CHARACTERIZATION OF LAYER-SPECIFIC MARKER ORTHOLOGS OF THE TURTLE}

When we first cloned turtle cDNAs, the genomic sequence of the turtle was not available. Thus, we amplified partial fragments of orthologous cDNAs of the six mammalian layer-specific markers, Satb2, Mef2c, Rorb, Er81, Fezf2, and Tbr1 (Figure 3), by reverse transcriptase-polymerase chain reaction (RT-PCR) using degenerate primers designed for the consensus sequences deduced from amniote genes (Table 1). To confirm whether the cloned turtle cDNAs indeed encoded the designated genes, we analyzed the cDNA sequences using BLASTN, and the NCBI mRNA database (Reference RNA sequences) to identify the most closely related sequences. This survey found the highest similarity for each clone to the expected ortholog of the chicken (Gallus gallus domesticus) or the green anole (Anolis carolinensis; Table 2). We next constructed phylogenic trees for these six isolated clones by incorporating sequence data from the human (Homo sapiens), mouse (Mus musculus), chicken, green anole, and frog (Xenopus tropicalis; Figure 3 ). The cDNA clones from the turtle were closely clustered with the designated orthologous members of these species and not with paralogs, confirming that they were indeed the turtle orthologs of the mammalian layer-specific marker genes (Figure 3). After the draft genome sequence of Chinese soft shell turtle was released recently (Wang et al., 2013), we confirmed that all the cloned cDNA sequences corresponded to those of the recorded transcripts in the database.

\section{DEVELOPMENTAL EXPRESSION OF LAYER-SPECIFIC MARKERS IN THE TURTLE PALLIUM}

We analyzed the expression patterns of the layer-specific markers in the developing turtle pallium and compared them with those in the chick pallium. The developmental stages of the turtle embryo were empirically matched with the chicken stages that display similar histological characteristics in the pallium, (Figures 4A,B, 5A,B, 6A,B, and 7A,B), such as the thickness of the VZ and the density of mitotic cells in the VZ. Correlation of the stages was also confirmed as appropriate based on the maximally shared transcriptome of the whole embryos (Wang et al., 2013). In addition to the six newly cloned turtle orthologs of layer-specific markers, we also used antibody markers for Foxp1, and Ctip2, which specifically recognize the turtle and chicken proteins (Suzuki et al., 2012). Among the eight layerspecific markers, we successfully detected the expression of seven marker genes: Tbr1, Er81, Fezf2, Mef2c, Satb2, Foxp1, and Ctip2, in the developing turtle pallium. Only the expression of Rorb, a layer 4 marker, was undetectable in the turtle pallium at all developmental stages examined, although this gene was weakly expressed in the chick mesopallium and nidopallium at E16 (data not shown).

When neurogenesis had just started in the pallium, at E12 in turtles and E5 in chicks, the layer 6 marker Tbr1, and the layer 5 marker Er81 were already expressed broadly by differentiated neurons in the pallium (Figures 4C-F). These expressions were not limited to the medial domain but stretched across the pallium, although Er81 was medially enriched in the chick pallium. Another layer 5 marker, Fezf2, was not expressed by postmitotic neurons in the pallium, but only detected in neural progenitors of the VZ, making a medial-high to lateral-low gradient, in both turtles and chicks (Figures $4 \mathbf{G}, \mathbf{H}$ ). None of the three layer $2 / 3$ markers were detected in the pallium at these early neurogenetic stages.

In slightly advanced mid-neurogenetic stages, E18 in turtles, and E7 in chicks, the layer 6 marker Tbr 1 was expressed by neurons in virtually all areas of the pallium in both species (Figures 5C,D). The expression level was more intense in the lateral side than in the medial side. The expression of the layer 5 marker Er81 was not detected in the turtle pallium at this stage (Figure 5E), but was detected in a small confined domain in the medial, superficial part of the chick pallium (Figure 5F) as reported previously (Nomura et al., 2008; Suzuki et al., 2012). At these stages, the expression of the layer 5 marker Fezf2 was no longer restricted to the VZ, but also expanded to include medially scattered neurons in the turtle and chick pallia. Fezf2 expression covered the Er81-expressing region in the chick pallium (Figures 5G,H). By these stages, two layer $2 / 3$ marker genes, Satb2, and Mef2c, started to be expressed in the lateral part of the pallium in both turtles and chicks (Figures 5I-L). Although the expression domains of the two genes largely overlapped, a closer look revealed that Mef2c expression slightly shifted to the marginal surface when compared to Satb2 expression in both species. The lateral domain commonly marked with the two layer $2 / 3$ genes was not completely separated from the medial domain marked with the layer 5 marker Fezf2, and there was substantial overlap between the Fezf2- and Satb2/Mef2c-positive domains in both turtles and chicks.

When neurogenesis is almost terminated, at E26 in turtles, and E10 in chicks, the layer 6 marker Tbr1 was still broadly expressed in the large, lateral part of the pallium in turtles and chicks (Figures 6C,D). The layer 5 marker Er81 was expressed in a very small restricted domain in the medial side of the pallium in both species (Figures 6E,F). These Er81-expressing medial domains of the two species were seemingly equivalent, but technically have been defined differently, as the turtle $\mathrm{mDC}$ and the chick APH. At these stages, the layer 5 marker Fezf2 reduced its expression in the VZ and was mainly expressed by differentiated neurons (Figures 6G,H). These Fezf2-expressing neurons showed a more confined distribution compared to earlier stages, but still covered a larger area than the Er81-expressing domain. More specifically, in the chick, signals for Fezf2 were most strongly concentrated in the Er81-expressing APH, and further spread weakly over the laterally positioned hyperpallium, and nidopallium. In the turtle, the signals spread more widely over the whole region of the DC, including into the Er81-expressing medial domain. From these 


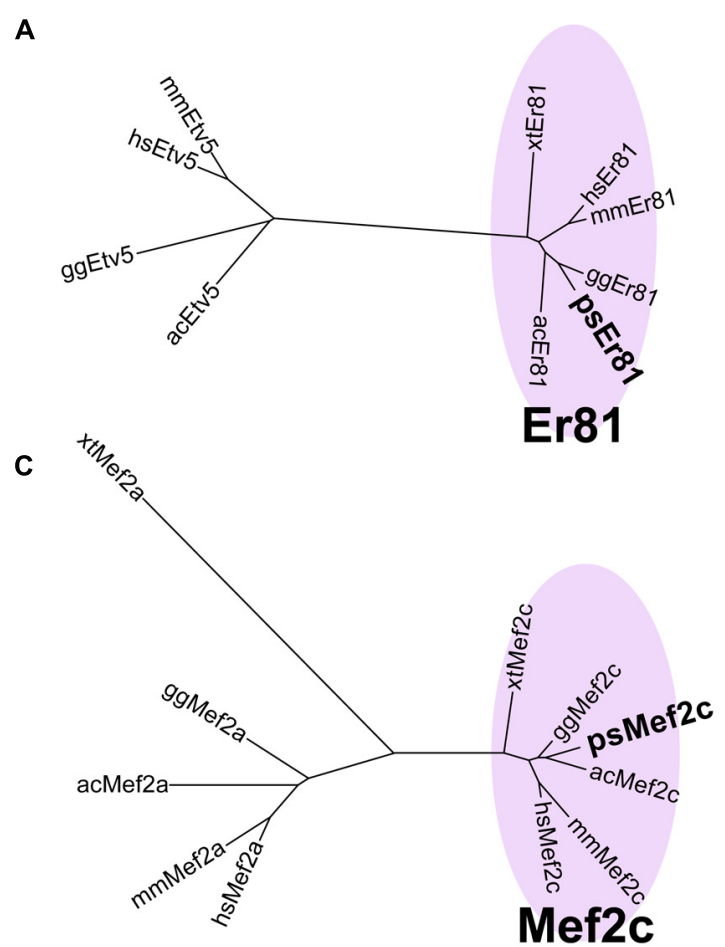

E

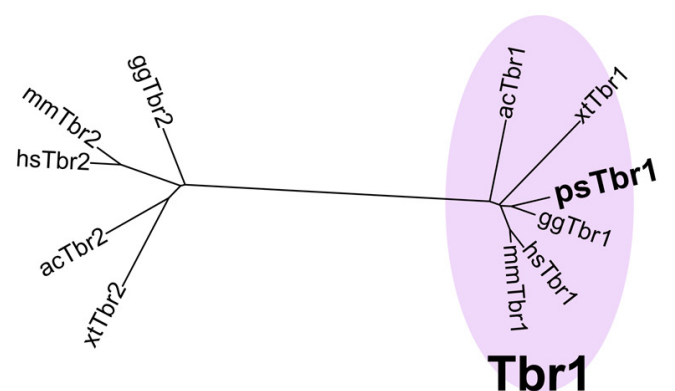

FIGURE 3 | Verification of layer-specific marker orthologs in the turtle. Molecular phylogenetic trees of (A) Er81, (B) Fezf2, (C) Mef2C, (D) Satb2, (E) Tbr1, and (F) Rorb cDNAs, constructed by the neighbor joining (NJ) method. Turtle cDNAs cloned in this study are indicated in boldface. In all six phylogenetic relationships, the turtle cDNAs are
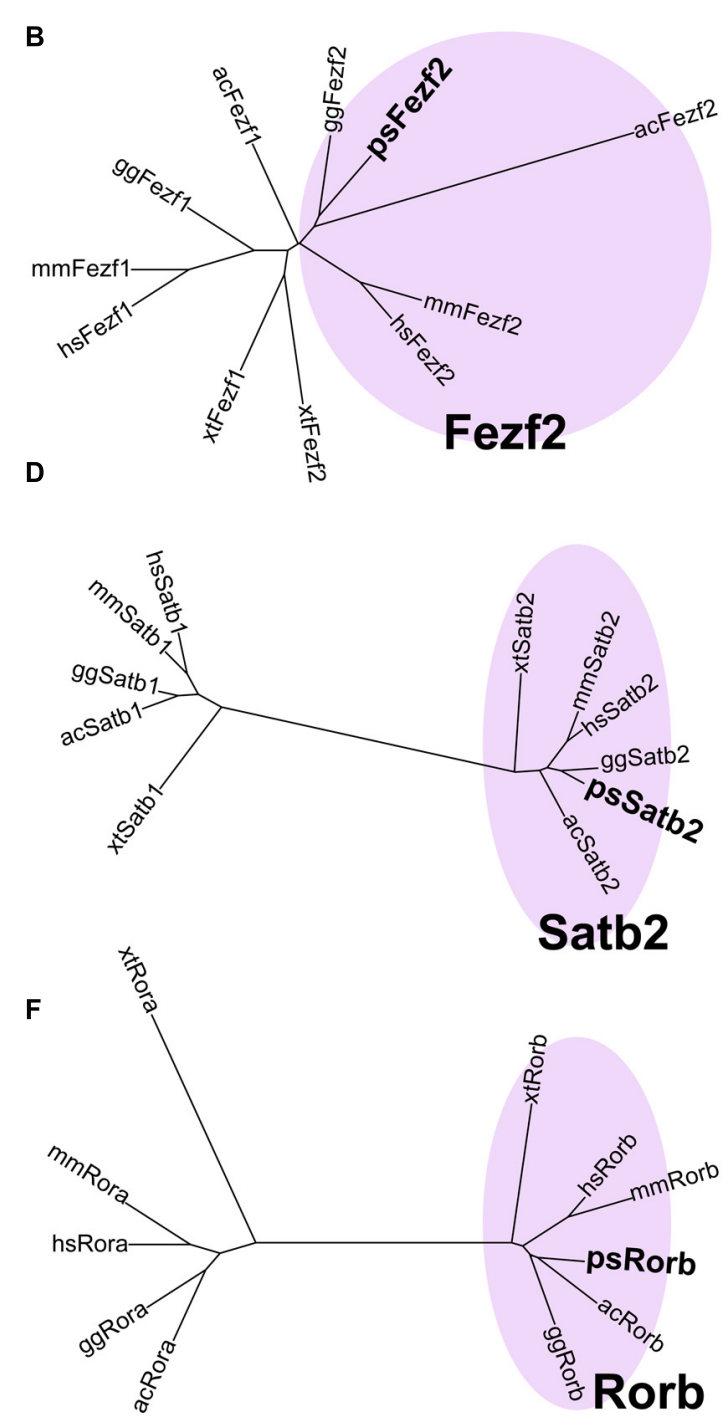

clustered with the orthologous genes (pink circled area) and separated from the paralogous genes. Abbreviations: ac, Anolis carolinensis (Green anole); gg, Gallus gallus (Chicken); hs, Homo sapiens (Human); mm, Mus musculus (Mouse); ps, Pelodiscus sinensis (Turtle); xt, Xenopus tropicalis (Frog). stages, the antibody markers started to show a specific labeling pattern (Figures $\mathbf{6 M}, \mathbf{N}$ ). In the chick pallium, as reported in the previous study (Suzuki et al., 2012), the layer 5 marker Ctip2 protein was expressed mainly in the APH and the dorsal part of the hyperpallium, which is presumably the future apical part of the hyper pallium (HA; green in Figure $6 \mathbf{N}$ ). The expression pattern was very similar to that of Fezf2, which is the transcriptional activator of Ctip2 in mammals (Chen et al., 2005, 2008; Molyneaux et al., 2005). In the turtle, Ctip2 was expressed more widely than Fezf2, and covered not only the DC but also other pallial areas, namely the PT and PC (green in Figure 6M). At these late developmental stages, three layer 2/3 marker genes, Mef2c, Satb2, and Foxp1, were all expressed in the lateral side of the pallium in turtles and chicks (Figures 6I-N). More specifically, in the turtle, the lateral compartment of the DC and PT were intensely labeled by all three markers, whereas the mesopallium and the neighboring densocellular part of the hyperpallium (HD) were labeled in the chick. In summary, the expression patterns of each layer-specific marker in the turtle and chick pallium resembled each other. A common trend observed in both species was that the layer 5 and the layer 2/3 markers were segregated in the medial and lateral domains, with a small overlap in the dorsal region of the pallium (Figures 60,P).

By the end of embryonic development, at E48 in turtles and E16 in chicks, many of the marker genes had restricted their expression domains. In the turtle, the expression of layer 6 marker Tbr1 was weakened, displaying a medial-low, lateralhigh gradient across the whole pallium (Figure $7 \mathrm{C}$ ). In the 


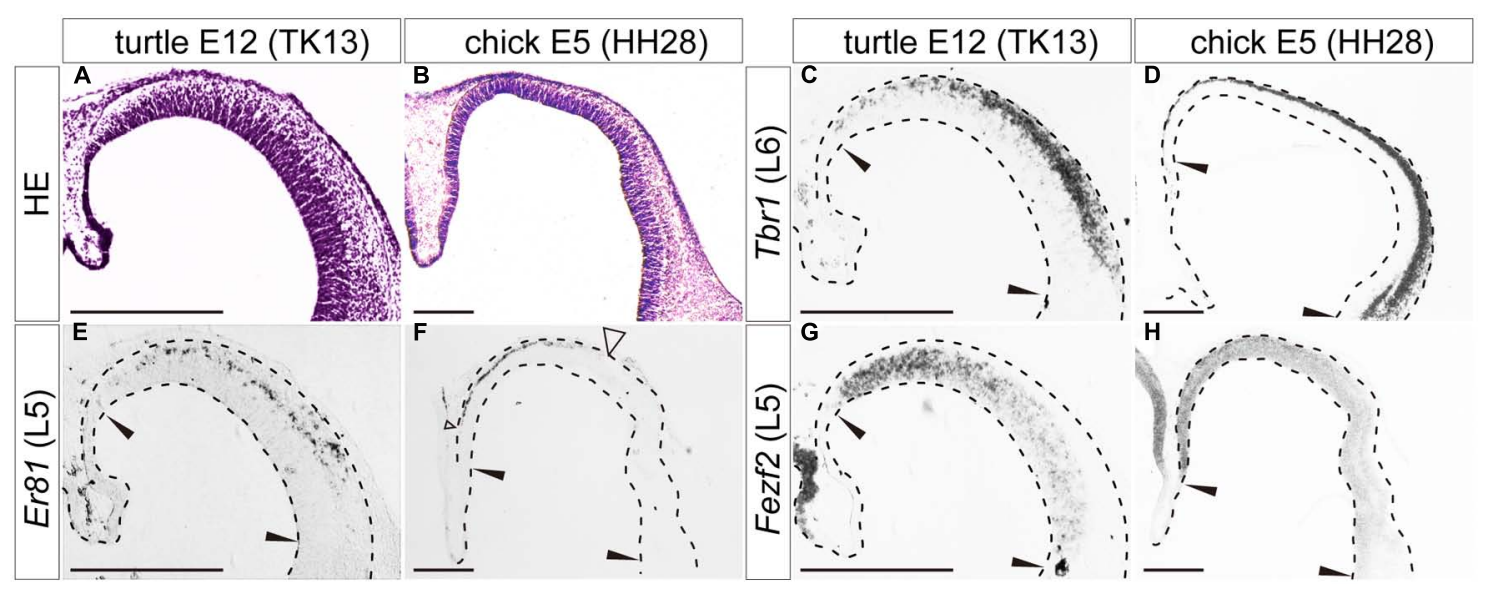

FIGURE 4 | Expression of layer-specific marker genes in the turtle and chick pallia at the early neurogenetic stage. Coronal sections of E12 turtle $(\mathbf{A}, \mathbf{C}, \mathbf{E}, \mathbf{G})$ and $\mathrm{E} 5$ chick $(\mathbf{B}, \mathbf{D}, \mathbf{F}, \mathbf{H})$ pallia, in which the mediolateral axis runs left to right and the dorsoventral axis runs top to bottom. (A,B) Hematoxylin and eosin (HE) staining of the pallium. (C,D) Expression patterns of Tbr1, a layer 6
(L6) marker. (E,F) Expression patterns of Er81, a layer 5 (L5) marker. An open arrowhead in $(\mathbf{F})$ indicates the lateral edge of the Er81-expression domain in the chick pallium. $(\mathbf{G}, \mathbf{H})$ Expression patterns of Fezf2, a layer 5 (L5) marker. Black arrowheads in each panel indicate the medial and lateral ends of the pallium. Scale bars: $250 \mu \mathrm{m}$. chick, Tbr1 only marked the ventral region of the pallium, including the mesopallium and nidopallium (Figure 7D). The expression of the layer 5 marker Er81 continued to be confined to the most medial compartment of the DC in the turtle and the APH in the chick (Figures 7E,F). The expression of the layer 5 marker Fezf2 became undetectable in the turtle pallium (Figure 7G). In the chick, Fezf2 expression was still weakly detected in the APH and HA, and in addition, was strongly expressed in the core nucleus of the caudodorsolateral pallium (CDLco) and nidopallium (Figure $7 \mathbf{H}$ ). The layer 5 marker Ctip2 was still widely expressed in the turtle pallium (Figure $7 \mathbf{M}$ ), whereas in the chick pallium, the expression was restricted to the Fezf2-positive domains (Figure $7 \mathbf{N}$ ). The three layer $2 / 3$ marker genes, Mef2c, Satb2, and Foxp1, continued to be expressed with significant overlap in the lateral domain of the pallium, the lateral part of DC (IDC), and PT in turtles, and the HD and mesopallium in chicks (Figures 6I-N). Similar to the slightly heterogeneous expressions of these genes reported in the chick (Suzuki et al., 2012), the spatial expression patterns of the layer 2/3 genes were also substantially different from each other in turtles; the expression of $\mathrm{Mef} 2 \mathrm{c}$ was mostly concentrated in the neuronal layer in the DC and PC, but not significantly detected in the internal nuclei, PT, and DVR (Figure 7I). In contrast, the expressions of Satb2 and Foxp1 were mainly detected in the PT (Figures 7K,M). Lastly, in these relatively mature stages, the overall expression domains of the layer 5 and layer 2/3 markers were more clearly segregated mediolaterally in the pallium of both turtles and chicks. The boundaries between the layer 5 and layer $2 / 3$ domains were positioned in the DC in the turtle, and in the hyperpallium in the chick (Figures 70,P).

\section{SIMILARITY OF THE NEUROGENETIC PATTERNS IN THE TURTLE AND CHICK PALLIA}

The spatiotemporal control of neurogenetic activities is a key factor that eventually creates distinct neuronal arrangements and morphological characteristics in different animal pallia (Suzuki et al., 2012; Aboitiz and Zamorano, 2013). We therefore examined neurogenetic activities in the turtle pallium and compared them to those in the chick. In the early stage of neurogenesis at E12, the mitotically active cells labeled by anti-phospho-histone $\mathrm{H} 3$ antibody were evenly distributed in the VZ throughout the pallium of the turtle, as observed in the chick at E5 (Figures 8A,B). In contrast, in the later stage at E18, the mitotically active cells were more abundantly observed in the lateral than in the medial side of the turtle pallium (Figure 8C). This laterally biased distribution of mitotic cells in the late phase of neurogenesis resembled that in the chick pallium at E8 (Figure 8D), in which it causes the late lateral expansion of the neuron population (Nomura et al., 2008; Suzuki et al., 2012). One minor difference between turtle and chick neurogenesis was that subventricular mitosis was lacking in the turtle pallium but existed in the chick pallium (Figures 8C',D'), as reported in a previous study (Cheung et al., 2007).

\section{DISCUSSION}

We studied turtle pallial development based on the expression patterns of eight layer-specific transcription factor genes, Tbr1, Er81, Fezf2, Ctip2, Rorb, Foxp1, Mef2c, and Satb2, and compared them with those in the chick pallium. We selected these genes not only as markers, but as potential determinants of neuronal fates, because many of them specified neuronal characteristics such as connection patterns in the rodent neocortex (Greig et al., 2013). The comparison indicated that the turtle and chick fundamentally share the spatial arrangement of molecularly defined neuron subtypes in the pallium. In both species, the layer 5, and layer $2 / 3$ layer-specific genes are expressed by neurons in the medial and lateral domains of the pallium, respectively. Furthermore, the turtle and chicken also shared spatiotemporal patterns of embryonic neurogenesis, leading to the expansion of late-born neurons in the lateral domain. Taken together, these results suggest that the developmental scheme of the turtle pallium is basically similar to 


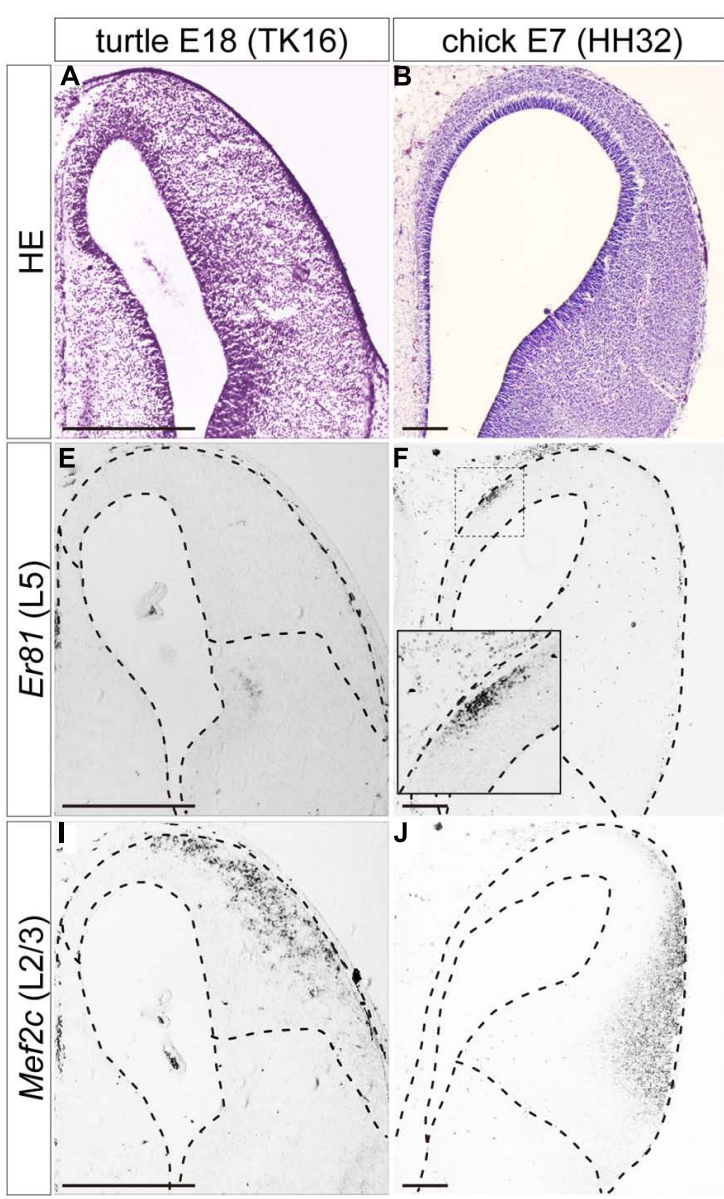

FIGURE 5 | Expressions of layer-specific marker genes in the turtle and chick pallia at the mid-neurogenetic stage. Coronal sections of E18 turtle $(\mathbf{A}, \mathbf{C}, \mathbf{E}, \mathbf{G}, \mathbf{I}, \mathbf{K})$ and $\mathrm{E7}$ chick $(\mathbf{B}, \mathbf{D}, \mathbf{F}, \mathbf{H}, \mathbf{J}, \mathbf{L})$ pallia, in which the

mediolateral axis runs left to right and the dorsoventral axis runs top to bottom. (A,B) HE staining of the pallium. (C) Tbr1 expression exhibits a medial-low, lateral-high gradient across the whole pallium of the turtle. (D)

Tbr1 expression covers the whole chick pallium except for the marginal and

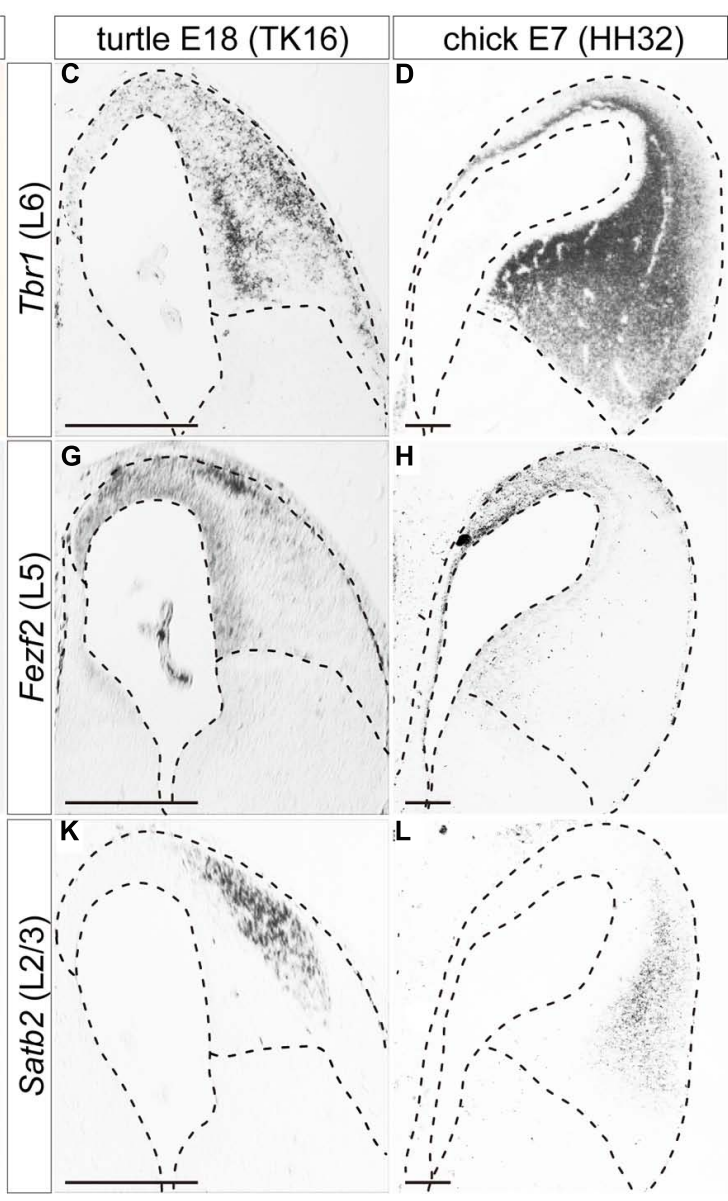

VZs. (E,F) Er81 is not expressed in the turtle pallium (E), but is expressed specifically in the medial part of the chick pallium (F). The inset is an enlargement of the rectangular box in the medial region. (G,H) Fezf2 expression exhibits a medial-high, lateral-low gradient in the VZ and differentiated neurons in both species. (I-L) Two layer 2/3 (L2/3) marker genes, Mef2c and Satb2, are expressed in the lateral region of the turtle and chick pallia. Scale bars: $250 \mu \mathrm{m}$. that of the chick pallium, and distinct from that of the mammalian neocortex.

\section{EXPRESSION OF LAYER-SPECIFIC TRANSCRIPTION FACTOR GENES Layer 6 marker, Tbr1}

The layer 6 marker Tbr1 gene was broadly expressed across the pallium in both turtles and chicks. This expression extensively overlapped with that of layer $2 / 3$ markers in the lateral part of the pallium. In certain developmental stages such as E18 in turtles and E7 in chicks (Figure 5), the layer 2/3 genes were more superficially expressed in the thin lateral domain, whereas Tbr1 was more widely expressed, as well as deeply, in the vicinity of the VZ. This configuration may give an impression of recapitulating the mammalian inside-out arrangement of layer 6 and 2/3 neurons. However, we consider it premature to discuss this possibility for the following reasons. First, we only analyzed one layer 6 marker in the analyses. Our and others' previous attempts to detect other layer 6 marker genes, such as Sox5, Foxp2, and Grg4, have failed to show their specific expressions in the chick pallium (Teramitsu et al., 2004; I.K.S. unpublished observation). Second, Tbr1 is not an exclusive marker for the neocortical layer 6, but is additionally expressed in other areas such as the neocortical layer $2 / 3$, the hippocampus and the olfactory cortex (Hevner et al., 2001; Englund et al., 2005). Therefore, we cannot be certain that the wide expression of Tbr1 in the turtle and chicken pallia in fact recapitulates the expression as the layer 6 marker. Finally, newly born neurons migrate roughly outside-in, but never inside-out, in the pallia of birds, and reptiles. Taken together, we consider the analogous counterparts of mammalian layer 6 neurons in chicks and turtles an open question.

\section{Layer 5 markers, Er81, Fezf2, and Ctip2}

We examined three markers expressed in layer 5 of the mammalian neocortex (Hashimoto et al., 2000; Matsuo-Takasaki et al., 2000; Arlotta et al., 2005; Yoneshima et al., 2006), Er81, Fezf2, and Ctip2. Among the three genes at least, Fezf2, and its downstream 


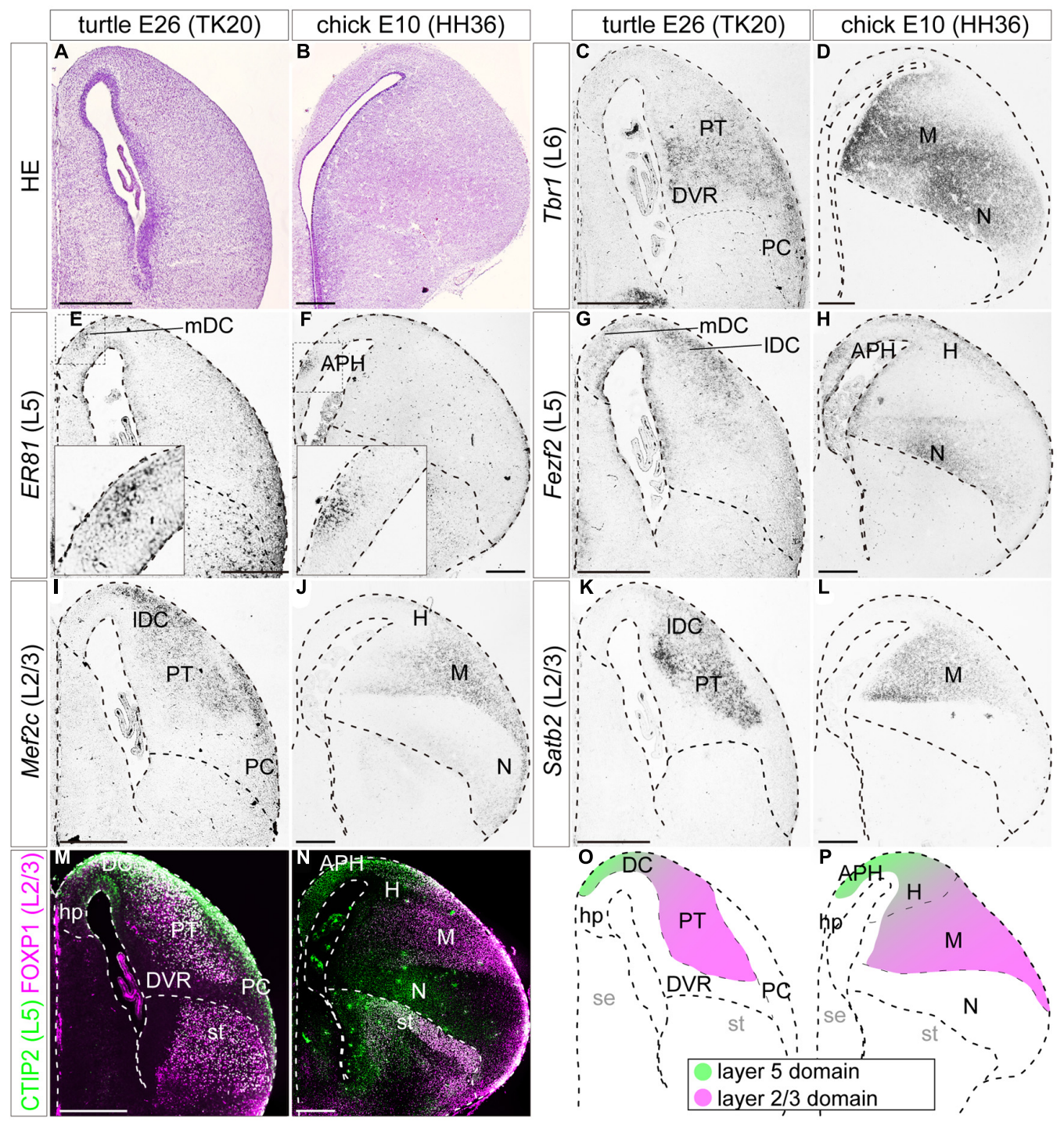

FIGURE 6 | Expressions of layer-specific marker genes in the turtle and chick pallia at the terminal neurogenetic stage. Coronal sections of E26 turtle $(\mathbf{A}, \mathbf{C}, \mathbf{E}, \mathbf{G}, \mathbf{I}, \mathbf{K}, \mathbf{M})$ and $\mathrm{E} 10$ chick $(\mathbf{B}, \mathbf{D}, \mathbf{F}, \mathbf{H}, \mathbf{J}, \mathbf{L}, \mathbf{N})$ pallia, in which the mediolateral axis runs left to right and the dorsoventral axis runs top to bottom. (A,B) Hematoxylin and eosin (HE) staining of the pallium.

(C,D) Tbr1 is enriched in the ventrolateral side of the turtle and chick pallia (E,F) Er81 is specifically localized in the small medial region of the turtle and chick pallia. The insets are enlargements of the dashed squares. (G) Fezf2 expression is widespread in the turtle DC, but does not expand to the other regions such as the PT, DVR, or PC. (H) In the chick, Fezf2 expression is concentrated in the $\mathrm{APH}$ and the ventral nidopallium, and weakly detected in the hyperpallium. Fezf2 and Er81 expressions are overlapped in the medial part of the pallium in both species. (I-L) Mef2c and Satb2 expressions are localized in the lateral part of the turtle and chick pallia. (M,N) Double immunostaining for Ctip2 (green) and Foxp1 (magenta). Ctip2 signals are broadly distributed across the turtle pallium including the DC, PT, and PC (M), whereas they are more specifically localized in the medial part of the chick pallium (N). Foxp1 is expressed in the turtle PT and the chick mesopallium. Scale bars: $250 \mu \mathrm{m}$.

$(\mathbf{O}, \mathbf{P})$ Schematic illustrations of the turtle and chick pallial subdivisions based on the expression patterns of seven layer-specific marker genes. Tbr1-expressing domain is not illustrated for simplicity. The distribution of Tbr1-expressing neurons is discussed later in the discussion section. Abbreviations: APH, parahippocampal region; DC, dorsal cortex; DVR, dorsal ventricular ridge; $H$, hyperpallium; hp, hippocampus (hippocampal homolog); IDC, lateral part of dorsal cortex; $M$, mesopallium; mDC, medial part of dorsal cortex; N, nidopallium; PC, piriform cortex; PT, pallial thickening; se, septum; st, striatum. target, Ctip2, are shown to be functionally crucial for providing the characteristics to mammalian layer 5 neocortical neurons and enabling them to project their axons to the brainstem (Chen et al., 2005, 2008; Molyneaux et al., 2005). All of these layer 5 genes were consistently expressed in the medial part of the turtle and chick pallium in an overlapping manner, leading to the assumption that these medial neurons have similar characteristics to the mammalian layer 5 neurons. Compared with the completely overlapping expressions of Fezf2 and Ctip2 in the mammalian, and chick pallia, Ctip2 was expressed more widely than Fezf2 in the turtle pallium. Therefore, in the turtle, the transcription of Ctip2 may be controlled by another regulator in addition to the 


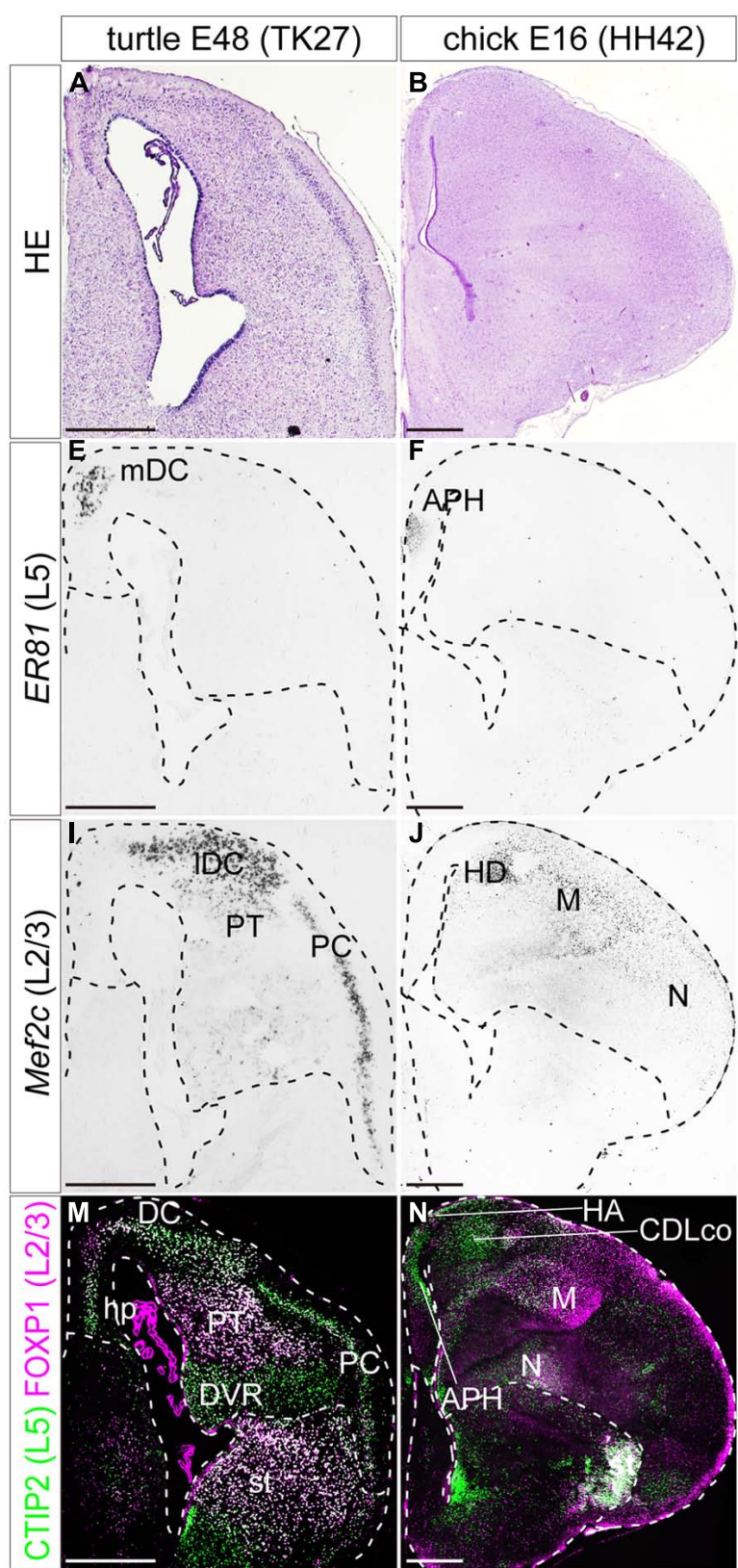

FIGURE 7|Expression of layer specific marker genes in the turtle and chick pallia at the maturation stage. Coronal sections of E48 turtle $(\mathbf{A}, \mathbf{C}, \mathbf{E}, \mathbf{G}, \mathbf{I}, \mathbf{K}, \mathbf{M})$ and $\mathrm{E} 16$ chick $(\mathbf{B}, \mathbf{D}, \mathbf{F}, \mathbf{H}, \mathbf{J}, \mathbf{L}, \mathbf{N})$ pallia, in which the mediolateral axis runs left to right and the dorsoventral axis runs top to bottom. (A,B) Hematoxylin and eosin (HE) staining of the pallium. (C) Tbr1 is expressed in virtually all the regions of the turtle pallium. (D) In the chick pallium, Tbr1 expression is detected in the ventrolateral regions, the mesopallium and the nidopallium. (E,F) Er81 expression is exclusively restricted to the small medial regions of the turtle and chick pallia. (G) Fezf2 is not expressed in the turtle at this stage. (H) In the chick, Fezf2 is weakly expressed in the medial regions of the pallium in addition to the strong expression in the CDLco and nidopallium. (I-L) Mef2c and Satb2 are expressed in the lateral part of the turtle and chick pallia. Mef2c is more

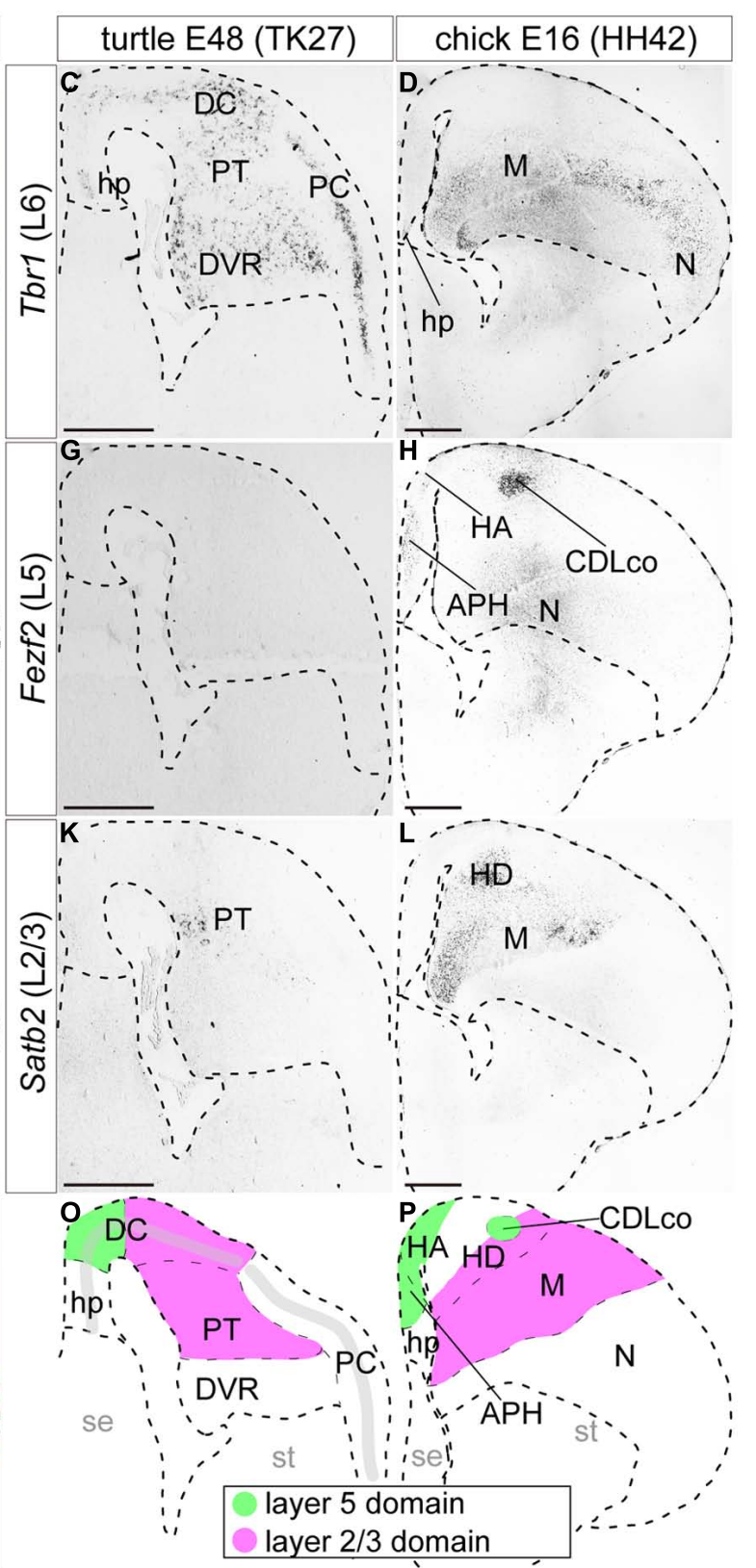

marginally expressed than Satb2. (M,N) Double immunostaining for Ctip2 (green) and Foxp1 (magenta). Ctip2 is expressed in virtually all areas in the turtle pallium (M), but is specifically confined to the medial part in the chick pallium (N). The expression of Foxp1 is confined to the lateral part of the turtle and chick pallia. Scale bars: $250 \mu \mathrm{m}$. (O,P) Schematic illustrations of the turtle and chick pallial subdivisions based on the expression patterns of the seven layer-specific marker genes. Abbreviations: $\mathrm{APH}$, parahippocampal region; CDLco, core nucleus of caudodorsolateral pallium; DC, dorsal cortex; DVR, dorsal ventricular ridge; $H$, hyperpallium; $H A$, apical part of the hyperpallium; $H D$, densocellular part of the hyperpallium; hp, hippocampus (hippocampal homolog); IDC, lateral part of the dorsal cortex; M, mesopallium; mDC, medial part of the dorsal cortex; N, nidopallium; PC, piriform cortex; PT, pallial thickening; se, septum; st, striatum. 


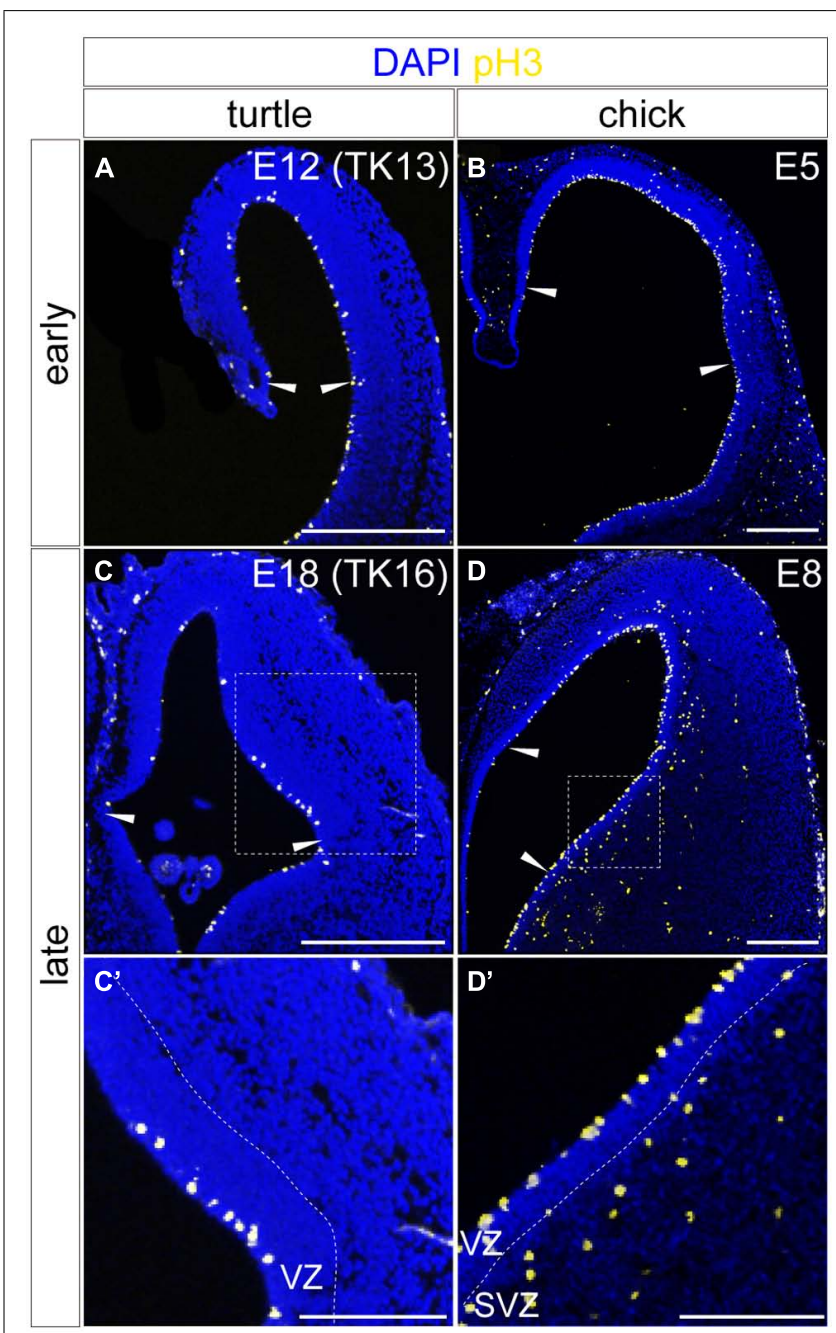

FIGURE 8 | Spatial distribution of mitotic cells in the developing turtle and chick pallia. Mitotic cells are labeled by anti-pH3 antibody in the turtle $(\mathbf{A}, \mathbf{C})$ and chick $(\mathbf{B}, \mathbf{D})$ pallia at early $(\mathbf{A}, \mathbf{B})$ and late $\mathbf{( C , D )}$ developmental stages; the mediolateral axis runs left to right and the dorsoventral axis runs top to bottom. Arrowheads indicate the medial and lateral borders of the pallium. White dotted insets in (C) and (D) are magnified in ( $\left.\mathbf{C}^{\prime}, \mathbf{D}^{\prime}\right)$. Scale bars: $250 \mu \mathrm{m}$ (A-D), $125 \mu \mathrm{m}$ (C',D').

upstream activator Fezf2. The expression patterns of these layer 5 marker genes observed in this study are consistent with the gene expression data recently reported in multiple reptile (Dugas-Ford et al., 2012; Nomura et al., 2013a) and avian species (Chen et al., 2013; Jarvis et al., 2013).

\section{Expression of layer 4 marker, Rorb}

Although we could not detect a specific expression of Rorb, recent reports have revealed its expression in the chicken and turtle pallia (Atoji and Karim, 2012; Dugas-Ford et al., 2012; Chen et al., 2013; Jarvis et al., 2013). Furthermore, Eag2, another layer 4 marker is also expressed in the same domains. Their shared expression suggests that the caudolateral DC of turtles and the part of hyperpallium and nidopallium entail neurons analogous to mammalian layer 4 neurons.

\section{Expression of layer 2/3 markers, Foxp1, Mef2c, and Satb2}

For this category, we examined three markers, Foxp1, Mef $2 c$, and Satb2, which are strongly expressed in layer 2/3 of the mammalian neocortex (Leifer et al., 1993; Ferland et al., 2003; Zhong et al., 2004; Britanova et al., 2005; Szemes et al., 2006; Hisaoka et al., 2010). Among these genes, Satb2 is critically important for providing intracortical projection identity to late-generated layer $2 / 3$ neurons in mice (Alcamo et al., 2008; Britanova et al., 2008). All of the layer 2/3 marker genes exhibited similar overlapping expressions in the lateral part of the turtle and chick pallia. Although the slight variation in expression patterns of individual genes suggested some heterogeneity in the neuronal population as seen in layer $2 / 3$ of mammalian neocortex, their consistent expressions in the same domain suggest that these neurons in the lateral domain are molecularly analogous to mammalian layer $2 / 3$ neurons.

\section{COMPARISON OF ARRANGEMENTS OF MOLECULARLY DEFINED NEURAL POPULATIONS IN THE PALLIA OF AMNIOTES}

In both turtle and chick pallia, the layer 5 and layer 2/3 markerexpressing neurons were segregated into the medial and lateral domains, respectively. Thus, the arrangements of these molecularly defined neuronal subtypes in the pallia are highly similar between the two distant sauropsid species, suggesting a common developmental plan for pallial neurogenesis in the sauropsids. More specifically, layer 5 marker-expressing neurons are localized in the medial DC of the turtle, and the APH, and apical part of the hyper pallium (HA) of the chick. In contrast, layer 2/3 markerexpressing neurons are localized in the lateral DC, and PT of the turtle, and in the densocellular part of the hyperpallium (HD), and mesopallium of the chick. Although the turtle DC and the chick hyperpallium have seemingly homogeneous histological features, they are actually subdivided into medial and lateral domains based on gene expression. This is also consistent with previous reports that demonstrate functional specialization of the medial and lateral domains of the turtle DC (Heller and Ulinski, 1987; Ulinski, 2007) and the chick hyperpallium (Butler and Hodos, 2005; Jarvis et al., 2005).

Given that these molecularly defined neuronal populations were sauropsid counterparts of mammalian layer-specific neurons, how can we reconcile the differences in the neuronal arrangement of sauropsids and mammals? We recently proposed that the spatiotemporal pattern of neurogenetic activities differentiates the chick pallium from that of mammals (Suzuki and Hirata, 2012, 2013; Suzuki et al., 2012). This hypothesis is based on our observation that chick pallial neural progenitors produce layer 5 and layer $2 / 3$ marker-expressing neurons in the same temporal sequence as that of mammalian progenitors in isolated culture conditions. This neocortex-like neurogenetic potential in chick neural progenitors is partially suppressed spatiotemporally in the chick pallium in vivo. Namely, neural progenitors on the medial side of the chick pallium precociously terminate neurogenesis before producing the late-born layer 2/3 marker-expressing neurons, the medial domain is thereby dominated by the early born layer 5 marker-expressing neurons. On the other hand, neural progenitors on the lateral side greatly expand their neurogenetic activities in the late developmental stage, and thereby accumulate the laterborn layer 2/3 marker-expressing neurons in the lateral domain 
(Suzuki et al., 2012). The conserved spatiotemporally biased neurogenetic activities in chick and turtle pallia (Figure 8) suggest that their common neurogenetic pattern creates similar medio-laterally separated neuronal arrangements in their pallia.

The mechanisms underlying this spatiotemporally biased neurogenesis remain unclear, but extrinsic factors in the in vivo environment are implicated (Suzuki etal., 2012; Suzuki and Hirata, 2013). In this regard, one candidate for the source is the ventral pallium (Puelles et al., 2000; Aboitiz and Zamorano, 2013). This region abuts the pallio-subpallio boundary (PSB), and in mammals functions as a signaling center, secreting morphogens and producing special cell types that regulate brain patterning, including Cajal-Retzius cells (Assimacopoulos et al., 2003; Bielle et al., 2005; Griveau et al., 2010; Teissier et al., 2010; Puelles, 2011). Interestingly, $D b x 1$, the transcription factor expressed specifically in this region of the mouse, is not expressed in the avian corresponding region. Supplementation of $D b x 1$ expression in this region of the quail greatly enhances the production of CajalRetzius cells (Nomura et al., 2008). These data suggest that this region provides potentially different environments in mammalian and sauropsid lineages (Molnár and Butler, 2002; Molnár et al., 2006; Puelles, 2011). In close proximity to this region is the DVR, which is another distinguishable feature between mammals and sauropsids including both turtles and birds. This anatomical feature could be another candidate for the source of different environmental factors between the two animal lineages.

\section{POTENTIAL RELATIONSHIPS WITH PREVIOUS HYPOTHESES FOR THE NEOCORTICAL EVOLUTION}

Many important hypotheses have been proposed for the evolution of the mammalian neocortex. However, continued debates still rage regarding its homologous counterpart in other animals. Many of the confusions stem from different usages of the term "homology” (Aboitiz, 1999; Molnar and Pollen, 2013; Medina et al., 2013). The term can refer to the same embryonic origin, gene expressions, neuronal connectivities, or other characteristics depending on the proposers. The situation becomes even more complicated because these characteristics belong to biological processes occurring at different levels, and therefore can either be totally independent of, or somewhat related to, each other even for a certain, small, brain domain. We will discuss our observations in relation to the previous hypotheses according to their individual criteria for homology.

\section{Embryonic origin}

The great diversity of pallial morphologies in animals can be reduced when brains are observed in the very early stages of their development. Indeed, the expression patterns of brain patterning genes in the VZ are highly similar even among distant animal groups, and are therefore highly comparable (Puelles et al., 2000; Murakami et al., 2005; Sugahara et al., 2013). According to this line of analysis, the vertebrate pallium is commonly subdivided into four regions, medial, dorsal, lateral, and ventral (Puelles et al., 2000). Among them, the origin of the mammalian neocortex is attributed to the Emx-positive dorsal pallium. Our analyses basically focused on this dorsal pallium compartment. In addition, the medial end of the layer 5 marker-expressing domain such as the $\mathrm{mDC}$ in turtles and the APH in chicks probably includes descendants of the medial pallium (Nomura et al., 2008). It is possible that the division between the medial and dorsal pallia is not exclusive. Our previous analysis of neuron migration in the chick pallium did not support the idea of a boundary of cell migration between the two domains (Suzuki etal., 2012). Therefore, a mixed population of medial and dorsal pallial origins seem to constitute the layer 5 marker-expressing domain. On the other hand, tangential mixing of migrating neurons between the mesopallium and nidopallium has not been observed in previous studies (Nomura et al., 2008; Suzuki et al., 2012), suggesting totally distinct origins of the constituents in these two compartments. Because we did not focus on the Emx-negative nidopallium in chicks and the DVR in turtles, our results do not exclude the possibility that Emx-negative pallial domains contain neurons homologous to the mammalian neocortex judged by different criteria.

\section{Gene expression}

Gene expression analysis is a powerful approach to detect hidden similarities among regions. Our present study essentially relies on this analysis. Recently, two systematic expression analyses in avian brains have been conducted. One was an in situ hybridization study using 52 gene probes in eight avian species (Chen et al., 2013; Jarvis et al., 2013). Interestingly, the authors found similarity in the expression profiles between the HA and nidopallium, and between the $\mathrm{HD}$ and the mesopallium, which exhibited a "mirror image organization". The results of cell migration analyses (Nomura etal., 2008; Suzuki etal., 2012) do not support the common embryonic origin of these mirror-imaged domains. However, the similarity in gene expressions could reflect functional similarities in the domains. Of note is that our gene expression analyses of layer $2 / 3$ markers completely agree with this mirror-image model indicating a similarity between the HD and the mesopallium. The layer 5 marker Fezf2 also appreciably follows this rule of similar gene expressions in the HA and nidopallium.

The other systemic expression analysis was a comprehensive transcriptomic study that compared expression profiles of about 5000 highly expressed genes between adult chicken pallial domains and adult mouse neocortical layers (Belgard et al., 2013). The study did not detect significant similarity among any combinations of the avian pallial domains and the mammalian neocortical layers, except for a weak similarity between the avian nidopallium and the mammalian layer 4 . Because being unable to detect significant similarity does not preclude the possibility of homology, the interpretation of these results is not straightforward. For example, the functional importance of genes for evolution cannot be proportional to their expression levels. By analyzing many abundantly expressed genes together, the real significance of similarity in a minor fraction of genes may be buried. Likewise, mass collection of tissues for RNA sampling could conceal individual neuronal differences, even though most genes are in fact heterogeneously expressed by neurons in each pallial domain, as shown in our results. It will take time and effort to genuinely understand the meaning of comprehensive transcriptome data. 


\section{Connection patterns}

Another important criterion for considering homology of brain regions is neural connection patterns. One such pattern is the "nuclear-to-layered" hypothesis in the avian pallium that proposes one-by-one homologies between the avian pallial subdivisions (nuclei) and the mammalian neocortical layers (Karten, 1991; Jarvis et al., 2005; Jarvis, 2009). Our gene expression data concord well with the proposed avian circuits in the hyperpallium, previously called the wulst, which is analogous to the mammalian somatosensory and primary visual circuits. Under this hypothesis, the HA is regarded as an output component to project to the brainstem (Karten et al., 1973; Reiner and Karten, 1983; Watanabe et al., 1983; Veenman et al., 1995; Wild and Williams, 2000; Suzuki et al., 2012), and indeed this domain expressed the markers for mammalian layer 5 corticofugal neurons (Dugas-Ford et al., 2012; Suzuki et al., 2012). The HD and the mesopallium also expressed the layer 2/3 markers, just as expected (Bradley et al., 1985; Shimizu et al., 1995; Atoji and Wild, 2012; Suzuki et al., 2012). Moreover, other groups have provided evidence that the intermediate region between the layer 5 and 2/3-expressing domains indeed expresses layer 4 markers (Dugas-Ford et al., 2012), and have molecularly supported its function as the major recipient of thalamic axons (Karten et al., 1973). Therefore, the expression data of mammalian layer markers are well correlated with the organization of the proposed homologous connectivities in the avian hyperpallium and mammalian neocortex. Because our analyses focused on the Emxpositive dorsal pallium, the proposed homologous component for the auditory circuits in the nidopallium (Wang et al., 2010) is beyond the scope of our results.

The situation seems to slightly differ in turtles. Our preliminary axon labeling suggested the layer 5 marker-expressing domain in the turtle does not make descending connections to the brain stem, but instead the layer 2/3-expressing domain makes descending connections to the thalamus (Bradley et al., 1985; Shimizu et al., 1995; Medina and Reiner, 2000, I. K. S. unpublished observation). Thus, the connection patterns and gene expressions seem to be disconnected in the turtle. This is surprising because many of the layer-specific transcription factors used in this study are well known to regulate connection patterns of cortical neurons in rodents. These functions may not be universal among animal groups, and the downstream genes may be relatively easily changeable in different lineages. Such dissociation of categories for homology in some species may underlie the existence of many different hypotheses for reptilian brain evolution (Aboitiz et al., 2002).

\section{Neuronal birth order}

Our previous study has added a new aspect to considering homology. The key element for constructing the mammalian neocortex is birth order-dependency in specification of layer-specific neuronal subtypes (Temple, 2001; Okano and Temple, 2009). This is accomplished by each cortical progenitor that follows a stereotyped neurogenetic sequence so as to sequentially produce deep to upper subtypes (Shen et al., 2006; Gaspard et al., 2008). Our results showed that this birth order-dependent mechanism is not specific to mammals (Suzuki et al., 2012), because chick pallial neural progenitors recapitulate this mammalian-type neurogenetic sequence in culture. The in vivo chick neural progenitors also follow this mammalian-type sequence in a spatiotemporally restricted manner. Thus, the homology that we have observed in the previous study is likely to represent the birth order similarity of neurons. We suspect the same scenario is applicable to turtles based on the spatiotemporal neurogenetic patterns observed here. This new criterion for homology could be grounded by the shared neurogenetic mechanisms between mammals and sauropsids, and also by the consequent conservation of birth order-dependent regulation of their gene expressions. In this regard, this criterion significantly differed from those that have been conventionally used in comparative anatomical studies, and can thus provide a different dimension to the homology debate.

\section{POTENTIAL EVOLUTIONARY SCENARIOS OF THE AMNIOTE PALLIUM}

On the basis of the molecular and histological characteristics of the pallium in three distantly related amniotes, the turtles, birds, and mammals, we propose the following hypothetical scenario for pallial evolution (Figure 9). The last common ancestor of all three amniote groups already possessed multiple layer-specific neuron subtypes, generated by the conserved neurogenetic program (Suzuki and Hirata, 2012, 2013; Suzuki et al., 2012). The ancient conservation of neocortical subtypes in the stem ancestor of the amniotes is consistent with the preceding models (Karten, 1991, 2013) and distinct from more classical view of sequential addition of new neuronal types during the course of evolution toward the mammals (Marin-padilla, 1978). By spatiotemporally modifying the neurogenetic program, the last common ancestor of sauropsids, which existed around 277 million years ago (Wang et al., 2013), established a pallium in which the layer 5 and $2 / 3$ subtypes were mediolaterally separated. The highly elaborated DVR is another marked feature shared by the turtle and chicken, but not shared by the mammals, and therefore was also present in the pallium of the ancestral sauropsids but not in that of the ancestral mammals (Figure 9). Meanwhile, the ancestral mammals developed a layered neocortex by making full use of the same conserved neurogenetic program. The idea of distinct evolutionary origins of the neocortical neuron subtypes and the laminar distribution of them has been already proposed elsewhere (Karten, 2013, 1991). A remaining major question is spatial arrangement of layer-specific subtypes in the pallium of the amniote common ancestor. Future investigation of the amphibian pallium as an outgroup will pave the way for exploring the divergent process of the laminar architecture of the mammalian neocortex and the mediolaterally separated organization of the sauropsid pallium.

\section{ACKNOWLEDGMENTS}

We thank H. Nakamura and K. Shimamura for providing the chick Er81 and Tbr1 cDNA constructs, respectively. We are grateful to Drs. D. H. Tanaka, T. Gojobori, T. Kawasaki, T. Nomura, Y. Murakami and S. Aizawa for comments and encouragement. We thank Drs. A. Watanabe and M. Kawaguchi for providing the turtle materials. Ikuo K. Suzuki was an NIG Postdoctoral Research Fellow. This work was supported by Grants-in-Aid for Creative Scientific Research from the Japan Society for the Promotion of Science and the Center for the Promotion of Integrated Sciences (CPIS) of Sokendai. 


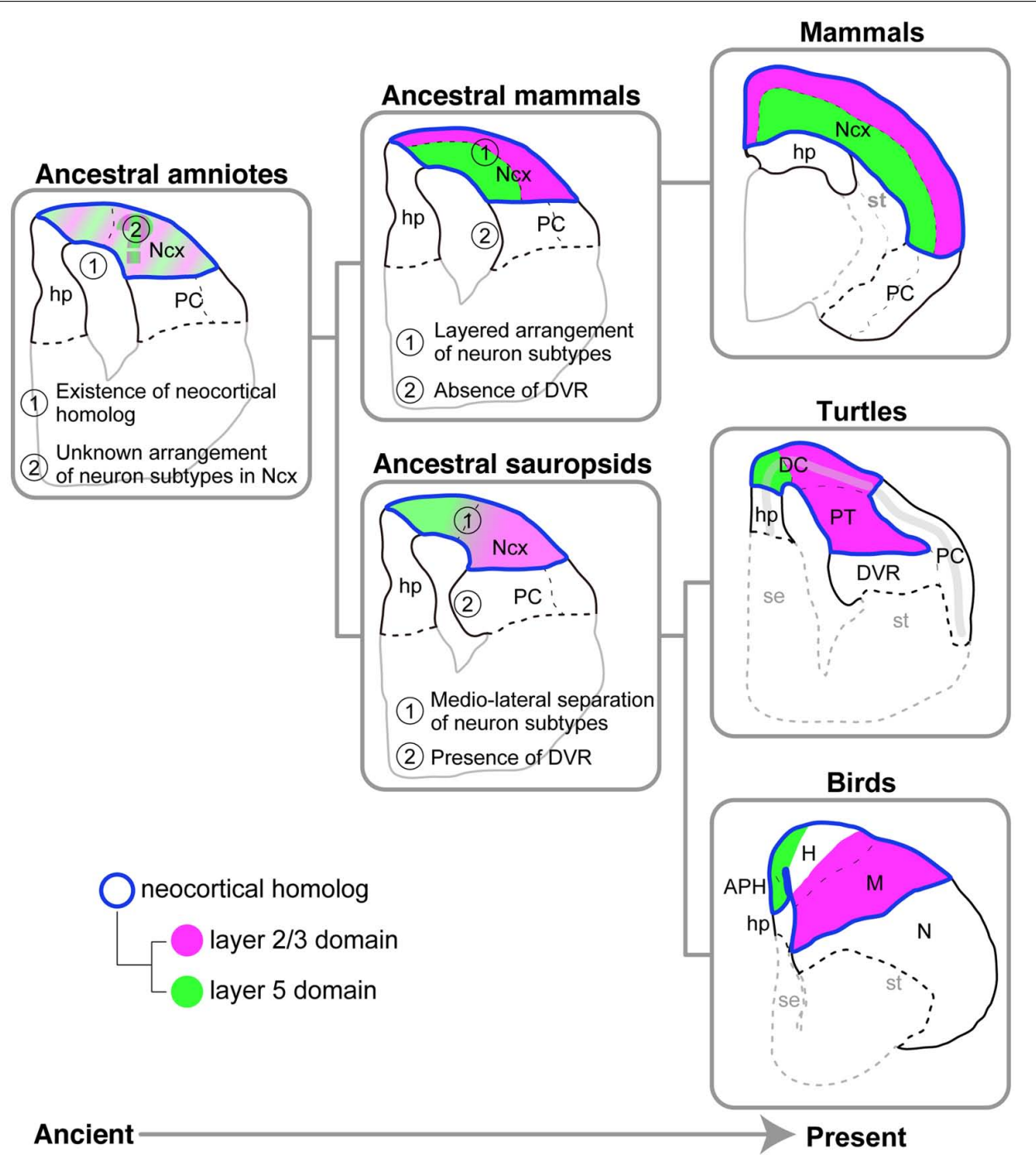

FIGURE 9 | Models for amniote pallial evolution. Evolutionary history flows from the ancient on the left side to the present on the right side. The pallia of the three living amniote groups are illustrated on the rightmost side. The hypothetical ancestral states deduced from the living species are illustrated on the center and left sides. The ancestral sauropsids, which are the common ancestors of the turtles and birds, are supposed to have had the following two characteristics shared by living turtles and birds: (1) separation of layer 5 and layer 2/3 subtypes in the medial and lateral domains of the pallium, respectively, and (2) the presence of DVR. The ancestral mammals, which are the common ancestors of all mammalian species, are supposed to have had the following two characteristics shared by all living mammalian species: (1) the layered arrangement of layer-specific neuron subtypes in the neocortex, and (2) absence of the
DVR. The ancestral amniotes, which are the common ancestors of all amniote species, are supposed to have had the following two characteristics shared by all living amniotes: (1) existence of a neocortical homolog ( $N c x$ ), and (2) existence of layer 5 and layer 2/3 subtype neurons in the neocortical homolog. It remains unclear whether this animal had a layered or mediolaterally separated arrangement of the layer subtypes in the neocortical homolog. Common color codes are used in all illustrations: Neocortical homolog (Ncx, encircled in blue), layer 2/3 neuron homolog (magenta), layer 5 neuron homolog (green). Abbreviations: APH, parahippocampal region; DC, dorsal cortex; DVR, dorsal ventricular ridge; $\mathrm{H}$, hyperpallium; hp, hippocampus (hippocampal homolog); $M$, mesopallium; $N$, nidopallium; Ncx, neocortex (neocortical homolog); PC, piriform cortex; PT, pallial thickening; se, septum; st, striatum.

\section{REFERENCES}

Aboitiz, F. (1999). Comparative development of the mammalian isocortex and the reptilian dorsal ventricular ridge. Evolutionary considerations. Cereb. Cortex 9 , 783-791. doi: 10.1093/cercor/9.8.783

Aboitiz, F., Montiel, J., Morales, D., and Concha, M. (2002). Evolutionary divergence of the reptilian and the mammalian brains: considerations on connectivity and development. Brain Res. Rev. 39, 141-153. doi: 10.1016/S0165-0173(02) 00180-7

Aboitiz, F., and Zamorano, F. (2013). Neural progenitors, patterning and ecology in neocortical origins. Front. Neuroanat. 7:38. doi: 10.3389/fnana.2013.00038
Alcamo, E. A., Chirivella, L., Dautzenberg, M., Dobreva, G., Fariñas, I., Grosschedl, R., et al. (2008). Satb2 regulates callosal projection neuron identity in the developing cerebral cortex. Neuron 57, 364-377. doi: 10.1016/j.neuron.2007.12.012

Arlotta, P., Molyneaux, B. J., Chen, J., Inoue, J., Kominami, R., and Macklis, J. D. (2005). Neuronal subtype-specific genes that control corticospinal motor neuron development in vivo. Neuron 45, 207-221. doi: 10.1016/j.neuron.2004. 12.036

Assimacopoulos, S., Grove, E. A., and Ragsdale, C. W. (2003). Identification of a Pax6-dependent epidermal growth factor family signaling source at the lateral edge of the embryonic cerebral cortex. J. Neurosci. 23, 6399-6403. 
Atoji, Y., and Karim, M. R. (2012). Expression of the neocortical marker, ROR $\beta$, in the entopallium and field L2 of adult chicken. Neurosci. Lett. 521, 119-124. doi: 10.1016/j.neulet.2012.05.068

Atoji, Y., and Wild, J. M. (2012). Afferent and efferent projections of the mesopallium in the pigeon (Columba livia). J. Comp. Neurol. 520, 717-741. doi: $10.1002 / \mathrm{cne} .22763$

Belgard, T. G., Montiel, J. F., Wang, W. Z., García-Moreno, F., Margulies, E. H., Ponting, C. P., et al. (2013). Adult pallium transcriptomes surprise in not reflecting predicted homologies across diverse chicken and mouse pallial sectors. Proc. Natl. Acad. Sci. U.S.A. 110, 13150-13155. doi: 10.1073/pnas.1307 444110

Bielle, F., Griveau, A., Narboux-Nême, N., Vigneau, S., Sigrist, M., Arber, S., et al. (2005). Multiple origins of Cajal-Retzius cells at the borders of the developing pallium. Nat. Neurosci. 8, 1002-1012. doi: 10.1038/nn1511

Blanton, M. G., and Kriegstein, A. R. (1991). Morphological differentiation of distinct neuronal classes in embryonic turtle cerebral cortex. J. Comp. Neurol. 310, 550-570. doi: 10.1002/cne.903100405

Bradley, P., Davies, D. C., and Horn, G. (1985). Connections of the hyperstriatum ventrale of the domestic chick (Gallus domesticus). J. Anat. 140, 577-589.

Britanova, O., Akopov, S., Lukyanov, S., Gruss, P., and Tarabykin, V. (2005). Novel transcription factor Satb2 interacts with matrix attachment region DNA elements in a tissue-specific manner and demonstrates cell-type-dependent expression in the developing mouse CNS. Neuroscience 21, 658-668. doi: 10.1111/j.1460-9568.2005.03897.x

Britanova, O., de Juan Romero, C., Cheung, A., Kwan, K. Y., Schwark, M., Gyorgy, A., et al. (2008). Satb2 is a postmitotic determinant for upper-layer neuron specification in the neocortex. Neuron 57, 378-392. doi: 10.1016/j.neuron.2007. 12.028

Butler, A. B., and Hodos, W. (2005). Comparative Vertebrate Neuroanatomy. 2nd Edn, New York: Wiley-Liss.

Carroll, R. L. (1988). Vertebrate Palaeontology and Evolution. New York: WH Freeman and Company.

Chen, B., Schaevitz, L. R., and McConnell, S. K. (2005). Fezl regulates the differentiation and axon targeting of layer 5 subcortical projection neurons in cerebral cortex. Proc. Natl. Acad. Sci. U.S.A. 102, 17184-17189. doi: 10.1073/pnas.0508732102

Chen, B., Wang, S. S., Hattox, A. M., Rayburn, H., Nelson, S. B., and McConnell, S. K. (2008). The Fezf2-Ctip2 genetic pathway regulates the fate choice of subcortical projection neurons in the developing cerebral cortex. Proc. Natl. Acad. Sci. U.S.A. 105, 11382-11387. doi: 10.1073/pnas.0804918105

Chen, C.-C., Winkler, C. M., Pfenning, A. R., and Jarvis, E. D. (2013). Molecular profiling of the developing avian telencephalon: regional timing and brain subdivision continuities. J. Comp. Neurol. 521, 3666-3701. doi $10.1002 / \mathrm{cne} .23406$

Cheung, A. F. P., Pollen, A. A., Tavare, A., Deproto, J., and Molnár, Z. (2007) Comparative aspects of cortical neurogenesis in vertebrates. J. Anat. 211, 164-176. doi: 10.1111/j.1469-7580.2007.00769.x

Dugas-Ford, J., Rowell, J. J., and Ragsdale, C. W. (2012). Cell-type homologies and the origins of the neocortex. Proc. Natl. Acad. Sci. U.S.A. 109, 16974-16979. doi 10.1073/pnas.1204773109

Englund, C., Fink, A., Lau, C., Pham, D., Daza, R. A. M., Bulfone, A., et al. (2005). Pax6, Tbr2, and Tbr1 are expressed sequentially by radial glia, intermediate progenitor cells, and postmitotic neurons in developing neocortex. J. Neurosci. 25, 247-251. doi: 10.1523/JNEUROSCI.289904.2005

Ferland, R. J., Cherry, T. J., Preware, P. O., Morrisey, E. E., and Walsh, C. A. (2003). Characterization of Foxp2 and Foxp1 mRNA and protein in the developing and mature brain. J. Comp. Neurol. 460, 266-279. doi: 10.1002/cne. 10654

Fernandez, A. S., Pieau, C., Reperant, J., Boncinelli, E., Wassef, M., and Repérant, J. (1998). Expression of the Emx-1 and Dlx-1 homeobox genes define three molecularly distinct domains in the telencephalon of mouse, chick, turtle and frog embryos: implications for the evolution of telencephalic subdivisions in amniotes. Development 125, 2099-2111.

Gaspard, N., Bouschet, T., Hourez, R., Dimidschstein, J., Naeije, G., van den Ameele, J., et al. (2008). An intrinsic mechanism of corticogenesis from embryonic stem cells. Nature 455, 351-357. doi: 10.1038/nature07287
Goffinet, A. M. (1983). The embryonic development of the cortical plate in reptiles: a comparative study in Emys orbicularis and Lacerta agilis. J. Comp. Neurol. 215, 437-452. doi: 10.1002/cne.902150408

Greig, L. C., Woodworth, M. B., Galazo, M. J., Padmanabhan, H., and Macklis, J. D. (2013). Molecular logic of neocortical projection neuron specification, development and diversity. Nat. Rev. Neurosci. 14, 755-769. doi: 10.1038/nrn3586

Griveau, A., Borello, U., Causeret, F., Tissir, F., Boggetto, N., Karaz, S., et al. (2010). A novel role for Dbxl-derived Cajal-Retzius cells in early regionalization of the cerebral cortical neuroepithelium. PLoS Biol. 8:e1000440. doi: 10.1371/journal.pbio. 1000440

Hamburger, V., and Hamilton, H. L. (1951). A series of normal stages in the development of the chick embryo. J. Morphol. 88, 49-92. doi: 10.1002/jmor.1050 880104

Hashimoto, H., Yabe, T., Hirata, T., Shimizu, T., Bae, Y., Yamanaka, Y., et al. (2000). Expression of the zinc finger gene fez-like in zebrafish forebrain. Mech. Dev. 97, 191-195. doi: 10.1016/S0925-4773(00)00418-4

Hedges, S. B., Dudley, J., and Kumar, S. (2006). TimeTree: a public knowledgebase of divergence times among organisms. Bioinformatics 22, 2971-2972. doi: 10.1093/bioinformatics/btl505

Heller, S. B., and Ulinski, P. S. (1987). Morphology of geniculocortical axons in turtles of the genera Pseudemys and Chrysemys. Anat. Embryol. (Berl.) 175, 505515. doi: 10.1007/BF00309685

Hevner, R. F., Shi, L., Justice, N., Hsueh, Y., Sheng, M., Smiga, S., et al. (2001). Tbr1 regulates differentiation of the preplate and layer 6. Neuron 29, 353-366. doi: 10.1016/S0896-6273(01)00211-2

Hisaoka, T., Nakamura, Y., Senba, E., and Morikawa, Y. (2010). The forkhead transcription factors, Foxp 1 and Foxp2, identify different subpopulations of projection neurons in the mouse cerebral cortex. Neuroscience 166, 551-563. doi: 10.1016/j.neuroscience.2009.12.055

Iwabe, N., Hara, Y., Kumazawa, Y., Shibamoto, K., Saito, Y., Miyata, T., et al. (2004). Sister group relationship of turtles to the bird-crocodilian clade revealed by nuclear DNA - coded proteins. Mol. Biol. Evol. 22, 1-4. doi: 10.1093/molbev/msi075

Jarvis, E. D. (2009). Bird brain: evolution. Neuroscience 2, 209-215.

Jarvis, E. D., Gunturkun, O., Bruce, L., Csillag, A. A., Karten, H. J., Kuenzel, W., et al. (2005). Avian brains and a new understanding of vertebrate brain evolution. Nat. Rev. Neurosci. 6, 151-159. doi: 10.1038/nrn1606

Jarvis, E. D., Yu, J., Rivas, M. V., Horita, H., Feenders, G., Whitney, O., et al. (2013) Global view of the functional molecular organization of the avian cerebrum: mirror images and functional columns. J. Comp. Neurol. 521, 3614-3665. doi: $10.1002 / \mathrm{cne} .23404$

Johnston, J. B. (1915). The cell masses in the forebrain of the turtle, cistudo carolina. J. Comp. Neurol. 25, 393-468. doi: 10.1002/cne.900250502

Karten, H. J. (1991). Homology and evolutionary origins of the "Neocortex". Brain Behav. Evo. 38, 264-272. doi: 10.1159/000114393

Karten, H. J. (2013). Neocortical evolution: neuronal circuits arise independently of lamination. Curr. Biol. 23, R12-R15. doi: 10.1016/j.cub.2012.11.013

Karten, H. J., Hodos, W., Nauta, W. J. H., and Revzin, A. M. (1973). Neural connections of the "Visual Wulst" of the avian telencephalon. Experimental studies in the pigeon(Columba livia) and owl (Speotyto cuniculana). J. Comp. Neurol. 150, 253-278. doi: 10.1002/cne.901500303

Kumar, S., and Hedges, S. B. (1998). A molecular timescale for vertebrate evolution. Nature 392, 917-920. doi: 10.1086/420415

Kumazawa, Y., and Nishida, M. (1997). Complete mitochondrial DNA sequences of the green turtle and blue-tailed mole skink: statistical evidence for archosaurian affinity of turtles. Mol. Biol. Evol. 16, 784-792. doi: 10.1093/oxfordjournals.molbev.a026163

Leifer, D., Krainc, D., Yu, Y. T., McDermott, J., Breitbart, R. E., Heng, J., et al. (1993). MEF2C, a MADS/MEF2-family transcription factor expressed in a laminar distribution in cerebral cortex. Proc. Natl. Acad. Sci. U.S.A. 90, 1546-1550. doi: 10.1073/pnas.90.4.1546

Marin-padilla, M. (1978). Dual origin of the mammalian neocortex and evolution of the cortical plate. Anat. Embryol. (Berl.) 152, 109-126. doi: 10.1007/bf00 315920

Martínez-Marcos, A., Lanuza, E., and Halpern, M. (1999). Organization of the ophidian amygdala: chemosensory pathways to the hypothalamus. J. Comp. Neurol. 412, 51-68. doi: 10.1002/(SICI)1096-9861(19990913)412:1<51::AID$\mathrm{CNE} 4>3.0 . \mathrm{CO} ; 2-\mathrm{M}$ 
Matsuo-Takasaki, M., Lim, J. H., Beanan, M. J., Sato, S. M., and Sargent, T. D. (2000). Cloning and expression of a novel zinc finger gene, Fez, transcribed in the forebrain of Xenopus and mouse embryos. Mech. Dev. 93, 201-204. doi: 10.1016/S0925-4773(00)00264-1

Medina, L., Abellán, A., and Desfilis, E. (2013). A never-ending search for the evolutionary origin of the neocortex: rethinking the homology concept. Brain Behav. Evol. 81, 150-153. doi: 10.1159/000348282

Medina, L., and Reiner, A. (2000). Do birds possess homologues of mammalian primary visual, somatosensory and motor cortices? Trends Neurosci. 23, 1-12. doi: 10.1016/S0166-2236(99)01486-1

Molnár, Z., and Butler, A. B. (2002). The corticostriatal junction: a crucial region for forebrain development and evolution. Bioessays 24, 530-541. doi: 10.1002/bies. 10100

Molnár, Z., Métin, C., Stoykova, A., Tarabykin, V., Price, D. J., Francis, F., et al. (2006). Comparative aspects of cerebral cortical development. Eur. J. Neurosci. 23, 921-934. doi: 10.1111/j.1460-9568.2006.04611.x

Molnar, Z., and Pollen, A. (2013). How unique is the human neocortex? Development 141, 11-16. doi: 10.1242/dev.101279

Molyneaux, B. J., Arlotta, P., Hirata, T., Hibi, M., and Macklis, J. D. (2005). Fezl is required for the birth and specification of corticospinal motor neurons. Neuron 47, 817-831. doi: 10.1242/dev.101279

Molyneaux, B. J., Arlotta, P., Menezes, J. R. L., and Macklis, J. D. (2007). Neuronal subtype specification in the cerebral cortex. Nat. Rev. Neurosci. 8, 427-437. doi: $10.1038 / \mathrm{nrn} 2151$

Murakami, Y., Uchida, K., Rijli, F. M., and Kuratani, S. (2005). Evolution of the brain developmental plan: insights from agnathans. Dev. Biol. 280, 249-259. doi: 10.1016/j.ydbio.2005.02.008

Nomura, T., Gotoh, H., and Ono, K. (2013a). Changes in the regulation of cortical neurogenesis contribute to encephalization during amniote brain evolution. Nat. Commun. 4, 2206. doi: 10.1038/ncomms3206

Nomura, T., Kawaguchi, M., Ono, K., and Murakami, Y. (2013b). Reptiles: a new model for brain evo-devo research. J. Exp. Zool. B Mol. Dev. Evol. 320, 57-73. doi: 10.1002/jez.b.22484

Nomura, T., Takahashi, M., Hara, Y., and Osumi, N. (2008). Patterns of neurogenesis and amplitude of reelin expression are essential for making a mammalian-type cortex. PLoS ONE 3:e1454. doi: 10.1371/journal.pone.0001454

Northcutt, R. G., and Kaas, J. H. (1995). The emergence and evolution of mammalian neocortex. Trends Neurosci. 18, 373-379. doi: 10.1016/01662236(95)93932-n

Okano, H., and Temple, S. (2009). Cell types to order: temporal specification of CNS stem cells. Curr. Opin. Neurobiol. 19, 112-119. doi: 10.1016/j.conb.2009. 04.003

Powers, A. S., and Reiner, A. (1980). A stereotaxic atlas of the forebrain and midbrain of the eastern painted turtle (Chrysemys picta picta). J. Hirnforsch. 21, 125-159. doi: $10.2307 / 1440052$

Puelles, L. (2011). Pallio-pallial tangential migrations and growth signaling: new scenario for cortical evolution? Brain Behav. Evol. 78, 108-127. doi: $10.1159 / 000327905$

Puelles, L., Kuwana, E., Puelles, E., Bulfone, A., Shimamura, K., Keleher, J., et al. (2000). Pallial and subpallial derivatives in the embryonic chick and mouse telencephalon, traced by the expression of the genes Dlx-2, Emx-1, Nkx-2.1, Pax-6, and Tbr-1. J. Comp. Neurol. 424, 409-438. doi: 10.1002/10969861(20000828)424:3<409::AID-CNE3>3.0.CO;2-7

Redies, C., Medina, L., and Puelles, L. (2001). Cadherin expression by embryonic divisions and derived gray matter structures in the telencephalon. J. Comp. Neurol. 285, 253-285. doi: 10.1002/cne.1315

Reiner, A. J. (2000). A hypothesis as to the organization of cerebral cortex in the common amniote ancestor of modern reptiles and mammals. Novartis Found. Symp. 228, 83-102; discussion 102-113. doi: 10.1002/047084 6631.ch7

Reiner, A., and Karten, H. J. (1983). The laminar source of efferent projections from the avian Wulst. Brain Res. 275, 349-354. doi: 10.1016/0006-8993(83) 90996-4

Saitou, N., and Nei, M. (1987). The neighbor-joining method: a new method for reconstructing phylogenetic trees. Mol. Biol. Evol. 4, 406-425.
Shen, Q., Wang, Y., Dimos, J. T., Fasano, C. A., Phoenix, T. N., Lemischka, I. R., et al. (2006). The timing of cortical neurogenesis is encoded within lineages of individual progenitor cells. Nat. Neurosci. 9, 743-751. doi: 10.1038/ nn 1694

Shimizu, T., Cox, K., and Karten, H. J. (1995). Intratelencephalic projections of the visual wulst in pigeons (Columba livia). J. Comp. Neurol. 359, 551-572. doi: 10.1002/cne.903590404

Sugahara, F., Murakami, Y., Adachi, N., and Kuratani, S. (2013). Evolution of the regionalization and patterning of the vertebrate telencephalon: what can we learn from cyclostomes? Curr. Opin. Genet. Dev. 1-9. doi: 10.1016/j.gde.2013. 02.008

Sugiyama, S., and Nakamura, H. (2003). The role of Grg4 in tectal laminar formation. Development 130, 451-462. doi: 10.1242/dev.00232

Suzuki, I. K., and Hirata, T. (2012). Evolutionary conservation of neocortical neurogenetic program in the mammals and birds. Bioarchitecture 2, 1-6. doi: 10.4161/bioa.21032

Suzuki, I. K., and Hirata, T. (2013). Neocortical neurogenesis is not really "neo": a new evolutionary model derived from a comparative study of chick pallial development. Dev. Growth Differ. 55, 173-187. doi: 10.1111/dgd. 12020

Suzuki, I. K., Kawasaki, T., Gojobori, T., and Hirata, T. (2012). The temporal sequence of the mammalian neocortical neurogenetic program drives mediolateral pattern in the chick pallium. Dev. Cell 22, 863-870. doi: 10.1016/j.devcel.2012.01.004

Szemes, M., Gyorgy, A., Paweletz, C., Dobi, A., and Agoston, D. (2006). Isolation and characterization of SATB2, a novel AT-rich DNA binding protein expressed in development- and cell-specific manner in the rat brain. Neurochem. Res. 31, 237-246. doi: 10.1007/s11064-005-9012-8

Tamura, K., Peterson, D., Peterson, N., Stecher, G., Nei, M., and Kumar, S. (2011). MEGA5: molecular evolutionary genetics analysis using maximum likelihood, evolutionary distance, and maximum parsimony methods. Mol. Biol. Evol. 28, 2731-2739. doi: 10.1093/molbev/msr121

Teissier, A., Griveau, A., Vigier, L., Piolot, T., Borello, U., and Pierani, A. (2010). A novel transient glutamatergic population migrating from the pallial-subpallial boundary contributes to neocortical development. J. Neurosci. 30, 10563-10574. doi: 10.1523/JNEUROSCI.0776-10.2010

Temple, S. (2001). The development of neural stem cells. Nature 414, 112-117. doi: $10.1038 / 35102174$

Teramitsu, I., Kudo, L. C., London, S. E., Geschwind, D. H., and White, S. A. (2004). Parallel FoxP1 and FoxP2 expression in songbird and human brain predicts functional interaction. J. Neurosci. 24, 3152-3163. doi: 10.1523/JNEUROSCI.558903.2004

Tokita, M., and Kuratani, S. (2001). Normal embryonic stages of the chinese softshelled turtle Pelodiscus sinensis (Trionychidae). Zoolog. Sci. 18, 705-715. doi: 10.2108/zsj.18.705

Ulinski, P. (2007). "Visual cortex of turtles," in Evolution of Nervous Systems: A Comprehensive Reference, Vol. 2, Non-Mammalian Vertebrates, ed. John H. Kaas (Amsterdam: Elsevier), 195-203. doi: 10.1016/B0-12-370878-8/ 00133-6

Veenman, C. L., Wild, J. M., and Reiner, A. (1995). Organization of the avian "Corticostriatal" projection system: a retrograde and anterograde pathway tracing study in pigeons. J. Neurosci. 354, 87-126.

Wang, Y., Brzozowska-Prechtl, A., and Karten, H. J. (2010). Laminar and columnar auditory cortex in avian brain. Proc. Natl. Acad. Sci. U.S.A. 107, 12676-12681. doi: 10.1073/pnas.1006645107

Wang, Z., Pascual-Anaya, J., Zadissa, A., Li, W., Niimura, Y., Huang, Z., et al. (2013). The draft genomes of soft-shell turtle and green sea turtle yield insights into the development and evolution of the turtle-specific body plan. Nat. Genet. 45, 701-706. doi: 10.1038/ng.2615

Watanabe, M., Ito, H., and Masai, H. (1983). Cytoarchitecture and visual receptive neurons in the wulst of the Japanese quail (Coturnix coturnix japonica). J. Comp. Neurol. 213, 188-198. doi: 10.1002/cne.902130206

Wild, J. M., and Williams, M. N. (2000). Rostral wulst in passerine birds. I. Origin, course, and terminations of an avian pyramidal tract. J. Comp. Neurol. 450, 429-450. doi: 10.1002/(SICI)1096-9861(20000124)416:4<429::AIDCNE2>3.0.CO;2-X 
Yoneshima, H., Yamasaki, S., and Voelker, C. C. J. (2006). Er81 is expressed in a subpopulation of layer 5 neurons in rodent and primate neocortices. Neuroscience 137, 401-412. doi: 10.1016/j.neuroscience.2005.08.075

Zhong, Y., Takemoto, M., Fukuda, T., Avenue, Y., and York, N. (2004). Identification of the genes that are expressed in the upper layers of the neocortex. Cereb. Cortex 4, 1144-1152. doi: 10.1093/cercor/bhh074

Conflict of Interest Statement: The authors declare that the research was conducted in the absence of any commercial or financial relationships that could be construed as a potential conflict of interest.

Received: 16 January 2014; accepted: 20 March 2014; published online: 07 April 2014.
Citation: Suzuki IK and Hirata T (2014) A common developmental plan for neocortical gene-expressing neurons in the pallium of the domestic chicken Gallus gallus domesticus and the Chinese softshell turtle Pelodiscus sinensis. Front. Neuroanat. 8:20. doi: 10.3389/fnana.2014.00020

This article was submitted to the journal Frontiers in Neuroanatomy.

Copyright (c) 2014 Suzuki and Hirata. This is an open-access article distributed under the terms of the Creative Commons Attribution License (CC BY). The use, distribution or reproduction in other forums is permitted, provided the original author(s) or licensor are credited and that the original publication in this journal is cited, in accordance with accepted academic practice. No use, distribution or reproduction is permitted which does not comply with these terms. 